\title{
Probing doubly and singly charged Higgs bosons at the $p p$ collider HE-LHC
}

\author{
Rojalin Padhan $\odot,{ }^{*}$ Debottam Das, ${ }^{\dagger}$ Manimala Mitra, ${ }^{*}$ and Aruna Kumar Nayak ${ }^{\S}$ \\ Institute of Physics, Sachivalaya Marg, Bhubaneswar 751005, India and Homi Bhabha National Institute, \\ BARC Training School Complex, Anushakti Nagar, Mumbai 400094, India
}

(Received 17 October 2019; accepted 3 April 2020; published 29 April 2020)

\begin{abstract}
We analyze the signal sensitivity of multilepton final states at colliders that can arise from doubly and singly charged Higgs decay in a type-II seesaw framework. We assume the triplet vacuum expectation value to be very small and degenerate masses for both the charged Higgs states. The leptonic branching ratios of doubly and singly charged Higgs states have a large dependency on the neutrino oscillation parameters and the lightest neutrino mass scale, as well as the neutrino mass hierarchy. We explore this, as well as the relation between the leptonic branching ratios of the singly and doubly charged Higgs states, in detail. We evaluate the effect of these uncertainties on the production cross section of the multilepton signal. Finally, we present a detailed analysis of multilepton final states for a future hadron collider, HE-LHC, that can operate with center-of-mass energy $\sqrt{s}=27 \mathrm{TeV}$.
\end{abstract}

DOI: 10.1103/PhysRevD.101.075050

\section{INTRODUCTION}

The discovery of the Higgs boson at the Large Hadron Collider (LHC) has experimentally proven that fermions' and gauge bosons' masses in the Standard Model (SM) are generated via the Brout-Englert-Higgs (BEH) mechanism. However, one of the key questions that still remains unexplained is the origin of light neutrino masses and mixings. A number of neutrino oscillation experiments have observed that the solar and atmospheric neutrino mass splittings are $\Delta m_{12}^{2} \sim 10^{-5} \mathrm{eV}^{2}$ and $\Delta m_{13}^{2} \sim 10^{-3} \mathrm{eV}^{2}$, and the mixing angles are $\theta_{12} \sim 32^{\circ}, \theta_{23} \sim 45^{\circ}$, and $\theta_{13} \sim 9^{\circ}$ [1]. A Dirac mass term of the SM neutrinos can be generated by extending the SM to include right-handed neutrinos. However, this requires very small Yukawa couplings, that introduce an $\mathcal{O}\left(10^{-11}\right)$ order of magnitude hierarchy between SM fermion Yukawa couplings, and hence is unappealing. A different ansatz is that neutrinos are their own antiparticles, and hence their masses can have a different origin compared to the other SM fermions. One such profound mechanism is seesaw, where tiny $\mathrm{eV}$ masses of the Majorana neutrinos are generated from the leptonnumber-violating (LNV) $d=5$ operator $L L H H / \Lambda$ [2,3]. Being a higher-dimensional nonrenormalizable operator,

\footnotetext{
*rojalin.p@iopb.res.in

†debottam@iopb.res.in

manimala@iopb.res.in

\$nayak@iopb.res.in
}

Published by the American Physical Society under the terms of the Creative Commons Attribution 4.0 International license. Further distribution of this work must maintain attribution to the author(s) and the published article's title, journal citation, and DOI. Funded by SCOAP. there can be different UV completed theories behind this operator, commonly known as type-I, -II, and -III seesaw mechanisms. These models include extensions of the SM fermion/scalar contents by SM singlet fermions [4-10], $S U(2)_{L}$ triplet scalar bosons [11-14], and $S U(2)_{L}$ triplet fermions [15], respectively.

Among the above, the type-II seesaw model, where a triplet scalar field with the hypercharge $Y=+2$ is added to the SM, has an extended scalar sector. There are seven physical Higgs states that include singly and doubly charged Higgs states, and $C P$-even and -odd neutral Higgs states. The details of the Higgs spectra have been discussed in Refs. [16,17]. The neutral component of the triplet acquires a vacuum expectation value (VEV) $v_{\Delta}$, and it generates neutrino masses through the Yukawa interactions. The same Yukawa interaction between the lepton doublet and the triplet scalar field also dictates the charged Higgs phenomenology in this model. The presence of a doubly charged Higgs $\left(H^{ \pm \pm}\right)$ is the most appealing feature of this model, and hence, a discovery of this exotic particle will be a smoking gun signature of a type-II seesaw.

A number of searches have already been performed to search for the signatures of the doubly charged Higgs (see Ref. [18] for Tevatron, and Refs. [19-33] for LHC). Depending on the triplet VEV, the doubly charged Higgs boson can have distinct decay modes. For a low VEV, $v_{\Delta} \lesssim 10^{-4} \mathrm{GeV}$, this can decay into a same-sign dilepton, whereas for $v_{\Delta} \geq 10^{-4} \mathrm{GeV}$, it can decay to same-sign gauge bosons. For nondegenerate masses of doubly and singly charged Higgs states, another possible decay is the cascade decay of a doubly charged Higgs to a singly charged Higgs and SM states. This has been explored in Refs. [19-21]. The CMS and ATLAS Collaborations have 
searched for the same-sign dilepton final states with different flavors, and have excluded the mass of the doubly charged Higgs $\left(M_{H^{ \pm \pm}}\right)$below 820 and $870 \mathrm{GeV}$, respectively, at 95\% C.L. $[34,35]$. An alternative search, where the $H^{ \pm \pm}$is produced in association with two jets-i.e., vector boson fusion-gives relaxed constraints [36,37]. In another scenario, the doubly charged Higgs decays to same-sign $W^{ \pm}$boson pairs. The collider signatures and the discovery prospects of this scenario have been discussed in Refs. [38-40], and in Refs. [41,42]. The ATLAS Collaboration have searched for the same final state and have excluded the doubly charged Higgs mass between 200 and $220 \mathrm{GeV}$ at $95 \%$ C.L. [43]. Previous searches for $H^{ \pm \pm}$in the pair-production channel and their subsequent decays into same-sign leptons at LEP-II have set a constraint $M_{H^{ \pm \pm}}>97.3 \mathrm{GeV}$ at $95 \%$ C.L. [44]. For discussions on the Higgs triplet model at a linear collider, see Refs. [45-49], and for an $e p$ collider, see Ref. [50]. Displaced vertex signatures have been discussed in Refs. [33,51]. A review on this model is presented in Ref. [52].

While a number of searches at the LHC are ongoing to experimentally verify the presence of the doubly charged Higgs boson, in this work we explore the impact of light neutrino mass hierarchy and neutrino oscillation parameters, as well as the lightest neutrino mass scale $m_{0}$, on $H^{ \pm \pm}$ searches. We relate the branching ratios of doubly and singly charged Higgs decays for both normal and inverted mass hierarchy. We find that among the different leptonic modes, the decay mode of doubly charged Higgs into two same-sign electrons and the decay mode of a singly charged Higgs into an electron and neutrino are the least uncertain for inverted neutrino mass ordering, and have the potential to differentiate the neutrino mass hierarchy. We also discuss how the inclusion of uncertainties in the neutrino oscillation parameters affect the theory cross section, which may in turn change the mass limits of doubly charged Higgs in individual channels. As it is well known that for c.m. energy $\sqrt{s}=13$ (or 14) TeV LHC, the production of multi-TeV $H^{ \pm \pm}$will be difficult due to the suppressed cross section. However, by increasing c.m. energy one can probe heavier $H^{ \pm \pm}$. Therefore, we consider the pair production and associated production of the doubly charged Higgs boson and its subsequent decays into leptonic states, including taus, and analyze the discovery prospects of doubly charged Higgs at a future hadron collider (HE-LHC), that can operate with c.m. energy $\sqrt{s}=27 \mathrm{TeV}$. We consider both the three- and four-lepton final states, and present a detailed analysis taking into account different possible SM background processes. We find that in addition to the associated production, the pair production of doubly charged Higgs states also gives a significant contribution to the trilepton final states. We consider a wide range of doubly charged Higgs masses and explore the sensitivity reach with the projected luminosity $\left(15 \mathrm{ab}^{-1}\right)$ of HE-LHC [53,54].
Our paper is organized as follows: we briefly review the basics of the type-II seesaw model in Sec. II. In Sec. III, we discuss leptonic branching ratios of doubly charged $\left(H^{ \pm \pm}\right)$ and singly charged $\left(H^{ \pm}\right)$Higgs states, and the relation between $H^{ \pm \pm}$and $H^{ \pm}$decays. In Sec. IV, we discuss the effect of uncertainties in neutrino oscillation parameters on the production cross section of the multilepton signal. In Sec. V, we present the simulation of the multilepton signal at the $\sqrt{s}=27 \mathrm{TeV}$ LHC. Finally, we present our conclusions in Sec. VI.

\section{MODEL DESCRIPTION}

In this section, we briefly discuss the type-II seesaw model [11-14]. The model is based on the gauge group as of the SM gauge group, $G_{\mathrm{SM}}=S U(3)_{C} \times S U(2)_{L} \times$ $U(1)_{Y}$. Apart from the SM particles, the particle spectrum also contains one additional $S U(2)_{L}$ triplet scalar $\Delta$ with hypercharge $\mathrm{Y}_{\Delta}=+2$ :

$$
\Delta=\left(\begin{array}{cc}
\frac{\Delta^{+}}{\sqrt{2}} & \Delta^{++} \\
\frac{1}{\sqrt{2}}\left(v_{\Delta}+\delta^{0}+i \eta^{0}\right) & -\frac{\Delta^{+}}{\sqrt{2}}
\end{array}\right) .
$$

The SM Higgs doublet is represented as follows:

$$
\Phi=\left(\begin{array}{c}
\phi^{+} \\
\frac{1}{\sqrt{2}}\left(v_{\phi}+\phi^{0}+i \chi^{0}\right)
\end{array}\right) .
$$

After electroweak symmetry breaking, the neutral Higgs $\phi^{0}$ and $\delta^{0}$ acquire VEVs, denoted as $v_{\phi}$ and $v_{\Delta}$, respectively. The two VEVs satisfy $v^{2}=v_{\phi}^{2}+v_{\Delta}^{2}=(246 \mathrm{GeV})^{2}$. Below, we discuss different terms of the Lagrangian.

(1) The kinetic Lagrangian for the scalar sector is

$$
\mathcal{L}_{\text {kin }}=\left(\mathrm{D}_{\mu} \Phi\right)^{\dagger}\left(\mathrm{D}^{\mu} \Phi\right)+\operatorname{Tr}\left[\left(\mathrm{D}_{\mu} \Delta\right)^{\dagger}\left(\mathrm{D}^{\mu} \Delta\right)\right] .
$$

The covariant derivatives in Eq. (3) are defined as

$$
\begin{gathered}
\mathrm{D}_{\mu} \Phi=\partial_{\mu} \Phi+i \frac{\mathrm{g}}{2} \tau^{\mathrm{a}} \mathrm{W}_{\mu}^{\mathrm{a}} \Phi+\mathrm{ig}^{\prime} \frac{\mathrm{Y}_{\Phi}}{2} \mathrm{~B}_{\mu} \Phi, \\
\mathrm{D}_{\mu} \Delta=\partial_{\mu} \Delta+i \frac{\mathrm{g}}{2}\left[\tau^{\mathrm{a}} \mathrm{W}_{\mu}^{\mathrm{a}}, \Delta\right]+\operatorname{ig}^{\prime} \frac{\mathrm{Y}_{\Delta}}{2} \mathrm{~B}_{\mu} \Delta .
\end{gathered}
$$

Both $v_{\phi}$ and $v_{\Delta}$ contribute to the masses of weak gauge bosons at tree level. Therefore, the $\rho$ parameter $\left(=\frac{M_{W}^{2}}{M_{Z}^{2} \cos ^{2} \theta_{W}}\right)$ in this model is given by

$$
\rho=\frac{1+\frac{2 v_{\Delta}^{2}}{v_{\phi}^{2}}}{1+\frac{4 v_{\Delta}^{2}}{v_{\phi}^{2}}} .
$$


The current electroweak precision data [55] give the value of the $\rho$ parameter as $\rho=1.00037 \pm 0.00023$, which is $1.6 \sigma$ away from the tree-level SM prediction. We consider $2.18 \sigma$ experimental error on the measured central value of the $\rho$ parameter and estimate a conservative bound on $v_{\Delta}$, i.e., $v_{\Delta} \lesssim 2 \mathrm{GeV}$. Thus, the two VEVs satisfy $v_{\Delta} \ll v_{\phi}$.

(2) The Yukawa Lagrangian of this model is given by

$\mathcal{L}_{\mathrm{Y}}(\Phi, \Delta)=\mathcal{L}_{Y}^{\mathrm{SM}}(\Phi)+Y^{\nu} L_{L}^{T} C i \sigma_{2} \Delta L_{L}+$ H.c.

Here, the first term in $\mathcal{L}_{Y}(\Phi, \Delta)$ represents the Yukawa interactions of the SM Higgs doublet $(\Phi)$, and the second term is the needed Yukawa interaction of the triplet Higgs $(\Delta)$, that generates neutrino mass. $Y^{\nu}$ is the Yukawa coupling matrix, $C$ is the charge conjugation operator, and $\sigma_{2}$ is the Pauli matrix. $L_{L}$ is the left chiral lepton doublet. Once the triplet Higgs $(\Delta)$ acquires a vacuum expectation value $v_{\Delta}$, the second term in $\mathcal{L}_{Y}(\Phi, \Delta)$ generates a Majorana mass for the neutrino, which is given by

$$
M^{\nu}=\sqrt{2} Y^{\nu} v_{\Delta}
$$

In the above, $M^{\nu}$ is a complex symmetric $3 \times 3$ matrix, which can be diagonalized by an unitary transformation defined as $M^{\nu}=V_{\mathrm{PMNS}}^{*} m_{d}^{\nu} V_{\mathrm{PMNS}}^{\dagger}$. Here $m_{d}^{\nu}=\operatorname{diag}\left(m_{1}, m_{2}, m_{3}\right)$ is the diagonal light neutrino mass matrix, and $V_{\text {PMNS }}$ is the neutrino mixing matrix parametrized by the three mixing angles $\left(\theta_{12}, \theta_{13}, \theta_{23}\right)$ and three phases $\left(\phi_{1}, \phi_{2}, \delta\right)$.

(3) The scalar potential [16] with the two Higgs fields $\Phi$ and $\Delta$ is

$$
\begin{aligned}
V(\Phi, \Delta)= & m^{2} \Phi^{\dagger} \Phi+M^{2} \operatorname{Tr}\left(\Delta^{\dagger} \Delta\right) \\
& +\left(\mu \Phi^{T} \mathrm{i} \sigma_{2} \Delta^{\dagger} \Phi+\text { H.c. }\right)+\frac{\lambda}{4}\left(\Phi^{\dagger} \Phi\right)^{2} \\
& +\lambda_{1}\left(\Phi^{\dagger} \Phi\right) \operatorname{Tr}\left(\Delta^{\dagger} \Delta\right)+\lambda_{2}\left[\operatorname{Tr}\left(\Delta^{\dagger} \Delta\right)\right]^{2} \\
& +\lambda_{3} \operatorname{Tr}\left[\left(\Delta^{\dagger} \Delta\right)^{2}\right]+\lambda_{4} \Phi^{\dagger} \Delta \Delta^{\dagger} \Phi .
\end{aligned}
$$

All operators in the above scalar potential are selfconjugate except the operator containing $\mu$. Therefore, all parameters except $\mu$ are real. Although $\mu$ can pick up a would-be $C P$ phase, this phase is unphysical and can always be absorbed in a redefinition of the scalar fields. Together $Y^{\nu}$ and the $\mu$ term violate lepton number symmetry in this model. Minimization of $V(\Phi, \Delta)$ gives the following two conditions [16]:

$$
\begin{gathered}
M^{2}=\frac{2 \mu v_{\phi}^{2}-\sqrt{2}\left(\lambda_{1}+\lambda_{4}\right) v_{\phi}^{2} v_{\Delta}-2 \sqrt{2}\left(\lambda_{2}+\lambda_{3}\right) v_{\Delta}^{3}}{2 \sqrt{2} v_{\Delta}}, \\
m^{2}=\frac{\lambda v_{\phi}^{2}}{4}-\sqrt{2} \mu v_{\Delta}+\frac{\left(\lambda_{1}+\lambda_{4}\right) v_{\Delta}^{2}}{2} .
\end{gathered}
$$

Thus, the two mass parameters $m^{2}$ and $M^{2}$ can be eliminated, which leaves eight free parameters $\left(v_{\Delta}\right.$, $\left.v_{\phi}, \mu, \lambda, \lambda_{1}, \lambda_{2}, \lambda_{3}, \lambda_{4}\right)$. Further, $v^{2} \equiv v_{\Phi}^{2}+v_{\Delta}^{2}=$ $(246 \mathrm{GeV})^{2}$ reduces this set of free parameters down to seven. There are ten real scalar degrees of freedom present in this model, out of which three are the would-be Goldstone bosons, and they give masses to the SM weak gauge bosons after electroweak symmetry breaking. The remaining seven states are the physical Higgs bosons. Doubly charged scalars $\left[\Delta^{ \pm \pm}\left(\equiv H^{ \pm \pm}\right)\right]$are purely triplet, and are already in mass eigenbasis. The singly charged scalars $\left(\phi^{ \pm}\right.$, $\left.\Delta^{ \pm}\right)$and neutral scalars $\left(\chi^{0}, \eta^{0}, \phi^{0}, \delta^{0}\right)$ are not physical fields, as they share nontrivial mixings among them. We denote the mass eigenstates of the singly charged scalars by $G^{ \pm}$and $H^{ \pm}$, which are linear combinations of $\phi^{ \pm}$and $\Delta^{ \pm}$. Similarly, the two $C P$-odd physical fields are denoted by $G^{0}$ and $A$ (linear combinations of $\chi^{0}$ and $\eta^{0}$ ). The SM Higgs field $(h)$ and a heavy Higgs $(H)$ are the mass basis of the two neutral $C P$-even states $\phi^{0}$ and $\delta^{0} . G^{ \pm}$and $G^{0}$ are the three Goldstone bosons. These scalar mixings are small, as they are proportional to the triplet VEV $\left(v_{\Delta}\right)$. The presence of the doubly charged Higgs $\left(H^{ \pm \pm}\right)$is the unique feature of this model. For detailed discussion on the masses of these scalars and doublet-triplet mixing angles, see Ref. [16].

Assuming $v_{\Delta} \ll v_{\phi}$, the masses of the physical Higgs bosons are given by [16]

$$
\begin{aligned}
M_{H^{ \pm \pm}}^{2} & \simeq M_{\Delta}^{2}-\frac{\lambda_{4}}{2} v_{\phi}^{2}, \quad M_{H^{ \pm}}^{2} \simeq M_{\Delta}^{2}-\frac{\lambda_{4}}{4} v_{\phi}^{2}, \\
M_{h}^{2} & \simeq 2 v_{\phi}^{2} \lambda, \quad M_{H}^{2}=M_{A}^{2} \simeq M_{\Delta}^{2},
\end{aligned}
$$

where $M_{\Delta}^{2} \equiv \frac{\mu v_{\phi}^{2}}{\sqrt{2} v_{\Delta}}$. We identify the $h$ field as the neutral SM Higgs, with its mass denoted as $M_{h}$. The mass of the SM Higgs is primarily governed by $\lambda$. The parameter $M_{\Delta}$ determines the mass scale of all other Higgs bosons. Mass-squared differences between the scalars are given by

$M_{H^{ \pm}}^{2}-M_{H^{ \pm \pm}}^{2} \simeq \frac{\lambda_{4}}{2} v_{\phi}^{2}, \quad M_{H / A}^{2}-M_{H^{ \pm}}^{2} \simeq \frac{\lambda_{4}}{4} v_{\phi}^{2}$.

Note that the quartic coupling $\lambda_{4}$ of the potential dictates the mass splitting between $H^{ \pm}-H^{ \pm \pm}$and 
$H(A)-H^{ \pm}$. These two mass-squared differences are of similar order. Taking into account the electroweak precision data [56], the mass splitting of the triplet Higgs is constrained as $\delta M<40 \mathrm{GeV}$ [20,57]. Therefore, the value of $\lambda_{4}$ defines three different mass spectra of the triplet Higgs:
(a) $\lambda_{4}=0$ (Degenerate scenario): $M_{H^{ \pm \pm}} \simeq M_{H^{ \pm}} \simeq$ $M_{H / A}$.
(b) $\lambda_{4}>0$ (Positive scenario): $M_{H^{ \pm \pm}}<M_{H^{ \pm}}<$ $M_{H / A}$.
(c) $\lambda_{4}<0$ (Negative scenario): $M_{H^{ \pm \pm}}>M_{H^{ \pm}}>$ $M_{H / A}$.

In our entire analysis, we assume the degenerate scenario for triplet Higgs masses, where all the triplet-like scalars have the same masses. The lightest neutral Higgs, that is primarily originated from the doublet $\Phi$, is considered as the SM-like Higgs. In the degenerate Scenario, one triplet Higgs will not be able to decay into another triplet Higgs and a gauge boson. Going beyond the degenerate scenario opens up a number of other decay possibilities, such as the cascade decays $\mathrm{H}^{++} \rightarrow \mathrm{H}^{+} W^{+\star}, \mathrm{H}^{+} \rightarrow H / A W^{+\star}$ in the negative scenario and $H^{+} \rightarrow H^{++} W^{-\star}, H / A \rightarrow H^{+} W^{-\star}$ in the positive scenario. As discussed in Ref. [23], these decays can be dominant if the mass differences between the charged Higgs states are sufficiently large, $\delta M>1 \mathrm{GeV}$. In other mass ranges, these are very suppressed. In the next section, we discuss the decay widths and branching ratios of different Higgs states, assuming a degenerate scenario. Therefore, cascade decay is not very relevant in our analysis.

\section{BRANCHING RATIOS OF $\boldsymbol{H}^{ \pm \pm}$AND $\boldsymbol{H}^{ \pm}$}

The decay properties of charged Higgs states in different $v_{\Delta}$ regions has been discussed extensively in the literature $[19,58]$. For $v_{\Delta}<10^{-4} \mathrm{GeV}$, the dominant decay channel of the doubly charged Higgs is $H^{ \pm \pm} \rightarrow l_{i}^{ \pm} l_{j}^{ \pm}$, and that of the singly charged Higgs is $H^{ \pm} \rightarrow l_{i}^{ \pm} \nu$, which is clear from Figs. 4 and 5 of Ref. [19]. In this region of $v_{\Delta}$, it will be possible to find out the correct neutrino mass ordering by measuring the leptonic branching ratios of the charged Higgs states [59]. Note that, in the leptonic channel, the same Yukawa coupling governs both the doubly charged and singly charged Higgs decays. Therefore, the leptonic decays of these two Higgs states are related. Below, we discuss the different decay channels and the relation between $H^{ \pm \pm}$and $H^{ \pm}$decays in detail.

\section{A. $\boldsymbol{H}^{ \pm \pm}$Decays}

Partial decay width of $H^{ \pm \pm}$to a pair of same-sign leptons [19] is given by

$$
\Gamma_{l_{i} l_{j}} \equiv \Gamma\left(H^{ \pm \pm} \rightarrow l_{i}^{ \pm} l_{j}^{ \pm}\right)=\frac{1}{4 \pi\left(1+\delta_{i j}\right)}\left|Y_{i j}^{\nu}\right|^{2} M_{H^{ \pm \pm}} .
$$

We consider $v_{\Delta}<10^{-4} \mathrm{GeV}$, and hence, $H^{ \pm \pm}$predominantly decays to leptonic final states. The decay branching ratio (BR) has the following form:

$\mathrm{BR}\left(H^{ \pm \pm} \rightarrow l_{i}^{ \pm} l_{j}^{ \pm}\right)=\frac{\Gamma_{l_{i} l_{j}}}{\sum_{k l} \Gamma_{l_{k} l_{l}}}=\frac{2}{\left(1+\delta_{i j}\right)} \frac{\left|Y_{i j}^{\nu}\right|^{2}}{\sum_{k l}\left|Y_{k l}^{\nu}\right|^{2}}$,

where

$$
\sum_{k l}\left|Y_{k l}^{\nu}\right|^{2}=\frac{1}{2 v_{\Delta}^{2}} \sum_{i} m_{i}^{2}
$$

In Fig. 1 , we plot the $\operatorname{BR}\left(H^{ \pm \pm} \rightarrow l_{i}^{ \pm} l_{j}^{ \pm}\right)$as a function of the lightest neutrino mass $\left(m_{0}\right)$ for both normal $(\mathrm{NH})$ and inverted (IH) mass hierarchy. Blue and red bands represent $\mathrm{IH}$ and $\mathrm{NH}$, respectively. Similar plots have already been presented in Refs. [19,58] considering the $3 \sigma$ range of neutrino mixing angles and mass square differences. But here we also vary all phases in between 0 and $2 \pi$, and we consider the current values of neutrino oscillation parameters [1], including nonzero $\theta_{13}$. Some notable points about these plots are as follows:

(1) $m_{0}>0.1 \mathrm{eV}$ represents the quasidegenerate neutrino mass spectrum, which is disallowed by cosmological data [60].

(2) For $m_{0}<0.1 \mathrm{eV}$, and for the modes $e^{ \pm} e^{ \pm}, e^{ \pm} \mu^{ \pm}$, $e^{ \pm} \tau^{ \pm}$, the maximum value of the branching ratio in IH is larger than that in NH. For the $\mu^{ \pm} \mu^{ \pm}, \mu^{ \pm} \tau^{ \pm}$, $\tau^{ \pm} \tau^{ \pm}$modes, it is the reverse. This behavior can be understood from Eqs. (16)-(18), which are the ratios between the maximum branching ratios in $\mathrm{IH}$ and $\mathrm{NH}$ for a given decay channel in the hierarchical regime with $m_{0} \approx 0$. The exact equations are presented in the Appendix.

$$
\begin{aligned}
& \frac{\mathrm{BR}^{\max }\left(H^{ \pm \pm} \rightarrow e^{ \pm} \mu^{ \pm}\right)_{\mathrm{IH}}}{\mathrm{BR}^{\max }\left(H^{ \pm \pm} \rightarrow e^{ \pm} \mu^{ \pm}\right)_{\mathrm{NH}}} \\
& \approx \frac{c_{13}^{2}\left(c_{12}^{2} s_{13} s_{23}+2 c_{23} c_{12} s_{12}-s_{12}^{2} s_{13} s_{23}\right)^{2}}{2\left(0.2 c_{12} c_{23} s_{12}+s_{13} s_{23}\left(0.2 s_{12}^{2}+1\right)\right)^{2}} \approx 6.6,
\end{aligned}
$$

$$
\frac{\mathrm{BR}^{\max }\left(H^{ \pm \pm} \rightarrow e^{ \pm} e^{ \pm}\right)_{\mathrm{IH}}}{\mathrm{BR}^{\max }\left(H^{ \pm \pm} \rightarrow e^{ \pm} e^{ \pm}\right)_{\mathrm{NH}}} \approx \frac{c_{13}^{4}}{2\left(0.2 c_{13}^{2} s_{12}^{2}+s_{13}^{2}\right)^{2}} \approx 50,
$$

$$
\begin{aligned}
& \frac{\mathrm{BR}^{\max }\left(H^{ \pm \pm} \rightarrow \mu^{ \pm} \mu^{ \pm}\right)_{\mathrm{IH}}}{\mathrm{BR}^{\max }\left(H^{ \pm \pm} \rightarrow \mu^{ \pm} \mu^{ \pm}\right)_{\mathrm{NH}}} \\
& \quad \approx \frac{c_{23}^{4}}{2\left(0.2 c_{12}^{2} c_{23}^{2}+c_{13}^{2} s_{23}^{2}\right)^{2}} \approx 0.45 .
\end{aligned}
$$

In the above, we consider the values of the oscillation parameters that maximize the numerator and 

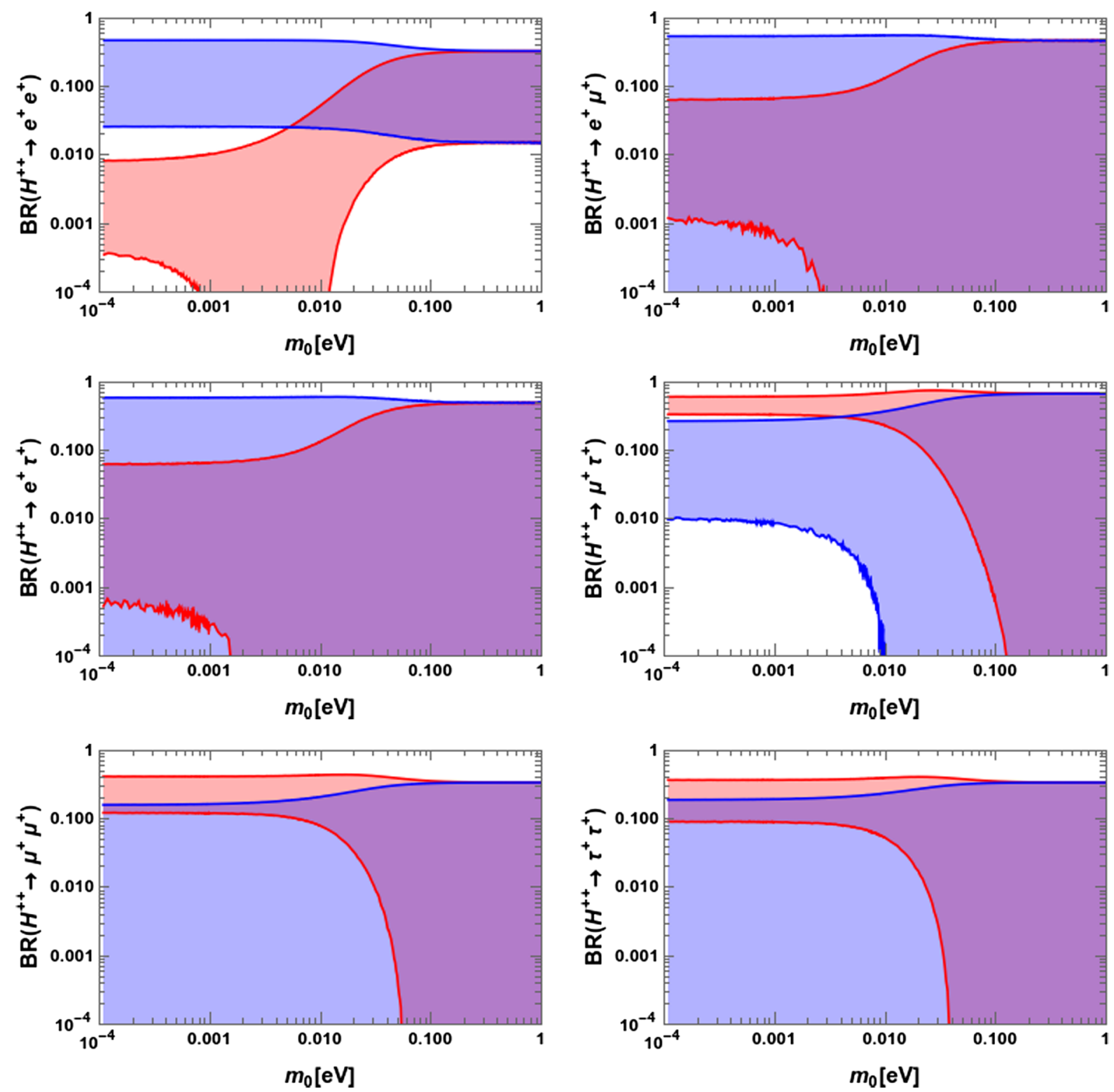

FIG. 1. Variation of branching ratios of $H^{++} \rightarrow l^{+} l^{+}$(where $l=e, \mu, \tau$ ) as a function of lightest neutrino mass $m_{0}$. [ $m_{0}$ is $m_{1}$ in normal hierarchy $(\mathrm{NH})$ and $m_{3}$ in inverted hierarchy (IH).] The band represents the uncertainty in the branching ratio due to $3 \sigma$ variation of neutrino oscillation parameters. We vary the $C P$ phases (Dirac and Majorana phases) in between 0 and $2 \pi$. The blue (red) band represents the $\mathrm{IH}(\mathrm{NH})$ pattern of neutrino mass.

denominator separately, as we are interested in the relative comparison of maximum branching ratios in $\mathrm{NH}$ and IH. The approximate expressions in the above equations clearly show that for IH neutrino mass spectrum, the $e^{ \pm} e^{ \pm}$and $e^{ \pm} \mu^{ \pm}$final states will be more favorable, as these channels can have large branching ratios. Although the final state $e^{ \pm} \tau^{ \pm}$has large branching ratio, further leptonic decays of $\tau^{ \pm}$will give suppression in cross section.

(3) There exists a large uncertainty in branching ratios that somewhat reduces for the choice of $C P$ phases to be zero. Among the different leptonic modes, $H^{ \pm \pm} \rightarrow e^{ \pm} e^{ \pm}$in IH is the most favorable mode for the entire range of $m_{0}$, as this decay mode has less uncertainty in the branching ratio, and there is a definite predicted lower value of $\operatorname{BR}\left(H^{ \pm \pm} \rightarrow e^{ \pm} e^{ \pm}\right)$. Irrespective of the value of the lightest neutrino mass, and the variation of oscillation parameters, the discovery of $H^{ \pm \pm}$will therefore be more favorable in this channel. An observation of $H^{ \pm \pm}$in any other leptonic decay mode except $e^{ \pm} e^{ \pm}$mode with a branching ratio limit $\operatorname{BR}\left(H^{ \pm \pm} \rightarrow e^{ \pm} e^{ \pm}\right)<0.015$ will indicate normal mass hierarchy in the light neutrino sector.

(4) Note that, except for $H^{ \pm \pm} \rightarrow e^{ \pm} e^{ \pm}$in $\mathrm{IH}$, all other decay modes heavily depend on the oscillation 
parameters and $m_{0}$. Moreover, for those decays, there may exist a cancellation region, in which the branching ratio becomes highly suppressed. This occurs when different terms in the partial decay widths cancel out each other. This is to note that, for $H^{ \pm \pm} \rightarrow e^{ \pm} e^{ \pm}$in $\mathrm{IH}$, such cancellation regions do not exist. As an example, the cancellation region for $H^{ \pm \pm} \rightarrow e^{ \pm} e^{ \pm}$in $\mathrm{NH}$, that exists within the range $10^{-3} \mathrm{eV} \lesssim m_{0} \lesssim$ $10^{-2} \mathrm{eV}$ can be explained as follows: For the choice $m_{1}=10^{-3} \mathrm{eV}$, the largest neutrino mass $\sum_{i} m_{i}^{2} \approx m_{3}^{2} \approx 4 \times 10^{-3} \mathrm{eV}^{2}$. Considering the $C P$ phases, $\phi_{1}=2 \delta-\phi_{2}=\pi$, one obtains

$$
\begin{aligned}
& \mathrm{BR}\left(H^{ \pm \pm} \rightarrow e^{ \pm} e^{ \pm}\right)_{\mathrm{NH}} \\
& \quad \approx 10^{-6} \frac{\left(-c_{12}^{2}+8 s_{12}^{2} c_{13}^{2}-60 s_{13}^{2}\right)^{2}}{4 \times 10^{-3}} \approx 10^{-4} .
\end{aligned}
$$

The branching ratio in IH is instead significantly large for the above choice of parameters. For similar values of $m_{0}=m_{3}=10^{-3} \mathrm{eV}, \phi_{1}, \phi_{2}$, and $\delta$ as mentioned in the case of $\mathrm{NH}$, one obtains

$$
\begin{aligned}
& \mathrm{BR}\left(H^{ \pm \pm} \rightarrow e^{ \pm} e^{ \pm}\right)_{\mathrm{IH}} \\
& \approx 10^{-6} \frac{\left(-60 c_{12}^{2}+60 s_{12}^{2} c_{13}^{2}-s_{13}^{2}\right)^{2}}{7 \times 10^{-3}} \approx 10^{-2} .
\end{aligned}
$$

(5) For the NH scenario, $H^{ \pm \pm} \rightarrow \mu^{ \pm} \mu^{ \pm} / \mu^{ \pm} \tau^{ \pm} / \tau^{ \pm} \tau^{ \pm}$ channels have the least uncertainty for $m_{0}<0.01 \mathrm{eV}$, and hence the discovery of $H^{ \pm \pm}$ in these above mentioned final states is more favorable for NH with $m_{0}<0.01 \mathrm{eV}$. Due to further decay of $\tau$ into leptonic states, which involves a smaller branching ratio, the overall cross section in the channel with $\tau$ will be relatively smaller than the channel with $\mu \mu$. Furthermore, a doubly charged Higgs cannot be fully reconstructed with the channel involving leptons from $\tau$, due to the presence of missing energy. Therefore, $H^{ \pm \pm} \rightarrow \mu^{ \pm} \mu^{ \pm}$decay mode will be more effective compared to the other two $H^{ \pm \pm} \rightarrow \mu^{ \pm} \tau^{ \pm} / \tau^{ \pm} \tau^{ \pm}$.

As we will discuss in Sec. IV, the variation of decay branching ratios of $H^{ \pm \pm}$with oscillation parameters, as well as the dependency on neutrino mass hierarchy have a large effect on the theory cross section of the four-lepton final states.

\section{B. $\boldsymbol{H}^{ \pm}$Decay}

$H^{ \pm}$decays predominantly to a lepton and neutrino for $v_{\Delta}<10^{-4} \mathrm{GeV}$. The partial decay width of $H^{ \pm}$to a lepton and neutrino [19] is given by

$$
\Gamma_{l_{j} \nu_{i}} \equiv \Gamma\left(H^{ \pm} \rightarrow l_{j}^{ \pm} \nu_{i}\right)=\frac{1}{16 \pi}\left|Y_{i j}^{+}\right|^{2} M_{H^{ \pm}} .
$$

In the above, $Y^{+}=\cos \theta^{+} \frac{m_{d}^{\nu} V_{P M N S}^{\dagger}}{v_{\Delta}}$, where $\theta^{+}$is the singly charged Higgs mixing angle. For $v_{\Delta}<10^{-4} \mathrm{GeV}$, the branching ratio for the decay $H^{ \pm} \rightarrow l_{j}^{ \pm} \nu_{i}$ is given by

$$
\operatorname{BR}\left(H^{ \pm} \rightarrow l_{j}^{ \pm} \nu_{i}\right)=\frac{\Gamma_{l_{j} \nu_{i}}}{\sum_{k l} \Gamma_{l_{k} \nu_{l}}}=\frac{\left|Y_{i j}^{+}\right|^{2}}{\sum_{k l}\left|Y_{k l}^{+}\right|^{2}},
$$

where

$$
\sum_{k l}\left|Y_{k l}^{+}\right|^{2}=\frac{\cos ^{2} \theta^{+}}{v_{\Delta}^{2}} \sum_{i} m_{i}^{2} .
$$

In Fig. 2, we plot $\operatorname{BR}\left(H^{ \pm} \rightarrow l_{j}^{ \pm} \nu\right) \equiv \sum_{i} \operatorname{BR}\left(H^{ \pm} \rightarrow\right.$ $\left.l_{j}^{ \pm} \nu_{i}\right)$ as a function of the lightest neutrino mass $m_{0}$, where we consider a $3 \sigma$ variation of neutrino oscillation parameters, and the variation of $C P$ phases between 0 and $2 \pi$. Important points to be noticed are as follows:

(1) Note that, for $m_{0}<0.1 \mathrm{eV}$ and for $\mathrm{IH}, H^{ \pm} \rightarrow e^{ \pm} \nu$ has a large branching ratio $(\sim 0.5)$. This decay channel, however, has a smaller branching ratio for NH. In the Appendix, this branching ratio has been calculated for $m_{0} \approx 0$. The maximum possible value of $\operatorname{BR}\left(H^{ \pm} \rightarrow e^{ \pm} \nu\right)$ in $\mathrm{IH}$ compared to that in $\mathrm{NH}$ is given by

$$
\frac{\mathrm{BR}^{\max }\left(H^{ \pm} \rightarrow e^{ \pm} \nu\right)_{\mathrm{IH}}}{\mathrm{BR}^{\max }\left(H^{ \pm} \rightarrow e^{ \pm} \nu\right)_{\mathrm{NH}}} \approx \frac{c_{13}^{2}}{2\left(0.04 c_{13}^{2} s_{12}^{2}+s_{13}^{2}\right)} \approx 13 .
$$

(2) Another important point to be noticed is that for $H^{ \pm}$, the uncertainty in the branching ratio is less compared to that for $H^{ \pm \pm}$. This occurs because the Yukawa couplings in the case of $H^{ \pm}$decay are independent of the two Majorana phases $\phi_{1}$ and $\phi_{2}$. This is evident from the equations given in the Appendix.

(3) Among the three decay modes of $H^{ \pm}, H^{ \pm} \rightarrow e^{ \pm} \nu$ has less uncertainty in the branching ratio, as the respective Yukawa is independent of Dirac $C P$ phase $\delta$ (see the Appendix). This branching ratio depends on $m_{0}$, $\theta_{12}, \theta_{13}$. The other two branching ratios for the muon and tau decay modes depend on $\theta_{23}$ and $\delta$ as well.

(4) The uncertainties in branching ratios for $H^{ \pm} \rightarrow \mu^{ \pm} \nu$ and $H^{ \pm} \rightarrow \tau^{ \pm} \nu$ are nearly equal. This is clear from the top-right and bottom plots of Fig. 2, where both the blue bands (in case of $\mathrm{IH}$ ) have similar spread. This feature also exists in the case of $\mathrm{NH}$.

Assuming 100\% branching ratios in leptonic decays, CMS and ATLAS searches have constrained $H^{ \pm \pm}$below $820 \mathrm{GeV}$ and $870 \mathrm{GeV}$, respectively. This is evident from the above discussion, that the branching ratio in any of the leptonic channels cannot reach up to $100 \%$. In Sec. IV, we reevaluate the production cross section of the four-lepton final state, originating from the pair production of doubly 

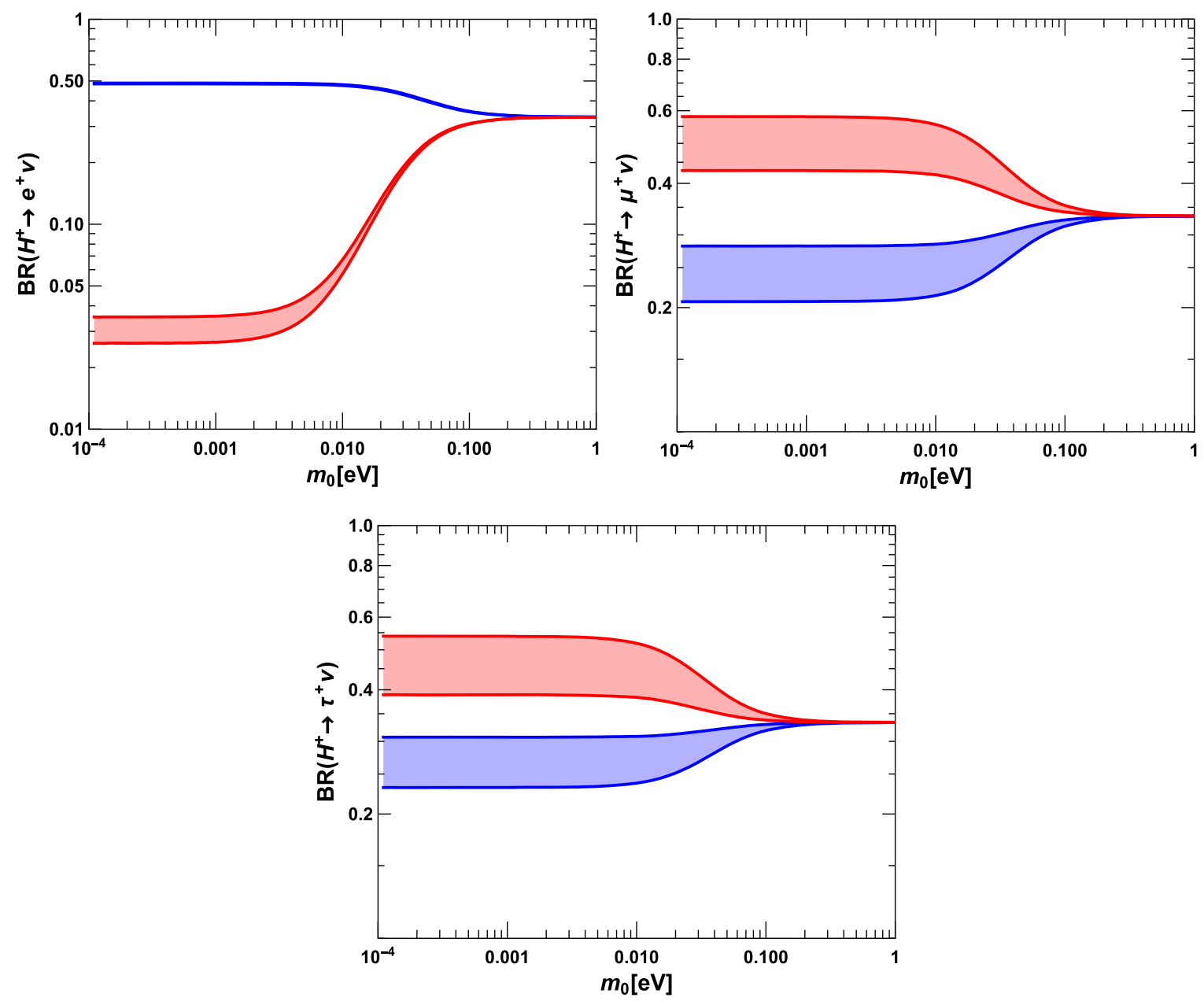

FIG. 2. Branching ratios of $H^{+} \rightarrow l^{+} \nu$ (where $l=e, \mu, \tau$ ). The blue (red) band represents the IH (NH) pattern of neutrino mass.

charged Higgs, for different leptonic channels, taking into account the uncertainties of branching ratios. As an example, we consider the decay channels $H^{ \pm \pm} \rightarrow e^{ \pm} e^{ \pm} /$ $e^{ \pm} \mu^{ \pm} / e^{ \pm} \tau^{ \pm}$in $\mathrm{IH}$, as they offer the largest branching ratio compared to $\mathrm{NH}$. Note that the maximum values of the branching ratio for the other three decay modes $\mu^{ \pm} \tau^{ \pm}$, $\mu^{ \pm} \mu^{ \pm}$, and $\tau^{ \pm} \tau^{ \pm}$are relatively smaller in IH. We provide a sample benchmark point in Table II, which shows that $e^{ \pm} \mu^{ \pm}$and $e^{ \pm} \tau^{ \pm}$have large branching ratios in $\mathrm{IH}$ as compared to the other modes. This is to clarify that the decay modes cannot simultaneously have maximum branching ratios. For the estimation in $\mathrm{NH}$, we assume the decay modes $H^{ \pm \pm} \rightarrow \mu^{ \pm} \tau^{ \pm} / \mu^{ \pm} \mu^{ \pm} / \tau^{ \pm} \tau^{ \pm}$, as they offer relatively large branching ratios.

\section{Relating $\boldsymbol{H}^{ \pm \pm}$and $\boldsymbol{H}^{ \pm}$decays}

The doubly charged Higgs $H^{ \pm \pm}$as well as singly charged Higgs $H^{ \pm}$interact with the leptons through the same Yukawa couplings that determine light neutrino masses. Therefore, the branching ratios of $H^{ \pm \pm}$into $l^{ \pm} l^{ \pm}$, and the branching ratio of $H^{ \pm}$into $l^{ \pm} \nu$ are related.
Figure 3 shows the variation of $H^{ \pm}$branching with $H^{ \pm \pm}$ branching for different leptonic decay channels. Here, we consider four illustrative samples of lightest neutrino masses $\left(m_{0}=0.0008,0.007,0.02,0.05 \mathrm{eV}\right)$ that cover almost the entire allowed light neutrino spectrum. Different color codes indicate different values of $m_{0}$. The spread of these color bands along the horizontal and vertical directions represents the uncertainty in $\operatorname{BR}\left(H^{ \pm \pm} \rightarrow l_{i}^{ \pm} l_{j}^{ \pm}\right)$and $\operatorname{BR}\left(H^{ \pm} \rightarrow l_{i}^{ \pm} \nu\right)$, respectively. In the upper-left panel of Fig. 3, we show the variation of $\mathrm{BR}\left(H^{ \pm \pm} \rightarrow e^{ \pm} e^{ \pm}\right)$with the variation of $\operatorname{BR}\left(H^{ \pm} \rightarrow e^{ \pm} \nu\right)$, where we assume IH for neutrino mass ordering. There is a small variation in $\operatorname{BR}\left(H^{ \pm} \rightarrow e^{ \pm} \nu\right)$ for a given value of $\mathrm{BR}\left(H^{ \pm \pm} \rightarrow e^{ \pm} e^{ \pm}\right)$that occurs due to the variation of oscillation parameters. The upper-right panel of Fig. 3 represents the variation of $\operatorname{BR}\left(H^{ \pm} \rightarrow e^{ \pm} \nu\right)$ with $\mathrm{BR}\left(H^{ \pm \pm} \rightarrow e^{ \pm} \mu^{ \pm}\right)$, again assuming $\mathrm{IH}$ as neutrino mass ordering. This also shows similar features to the previous plot. The plot in the lower panel in Fig. 3 shows the large variation of $\operatorname{BR}\left(H^{ \pm} \rightarrow \mu^{ \pm} \nu\right)$ with $\operatorname{BR}\left(H^{ \pm \pm} \rightarrow \mu^{ \pm} \mu^{ \pm}\right)$for NH. For smaller $m_{0}$, the $\operatorname{BR}\left(H^{ \pm \pm} \rightarrow \mu^{ \pm} \nu\right)$ has a large dependency on oscillation parameters. 

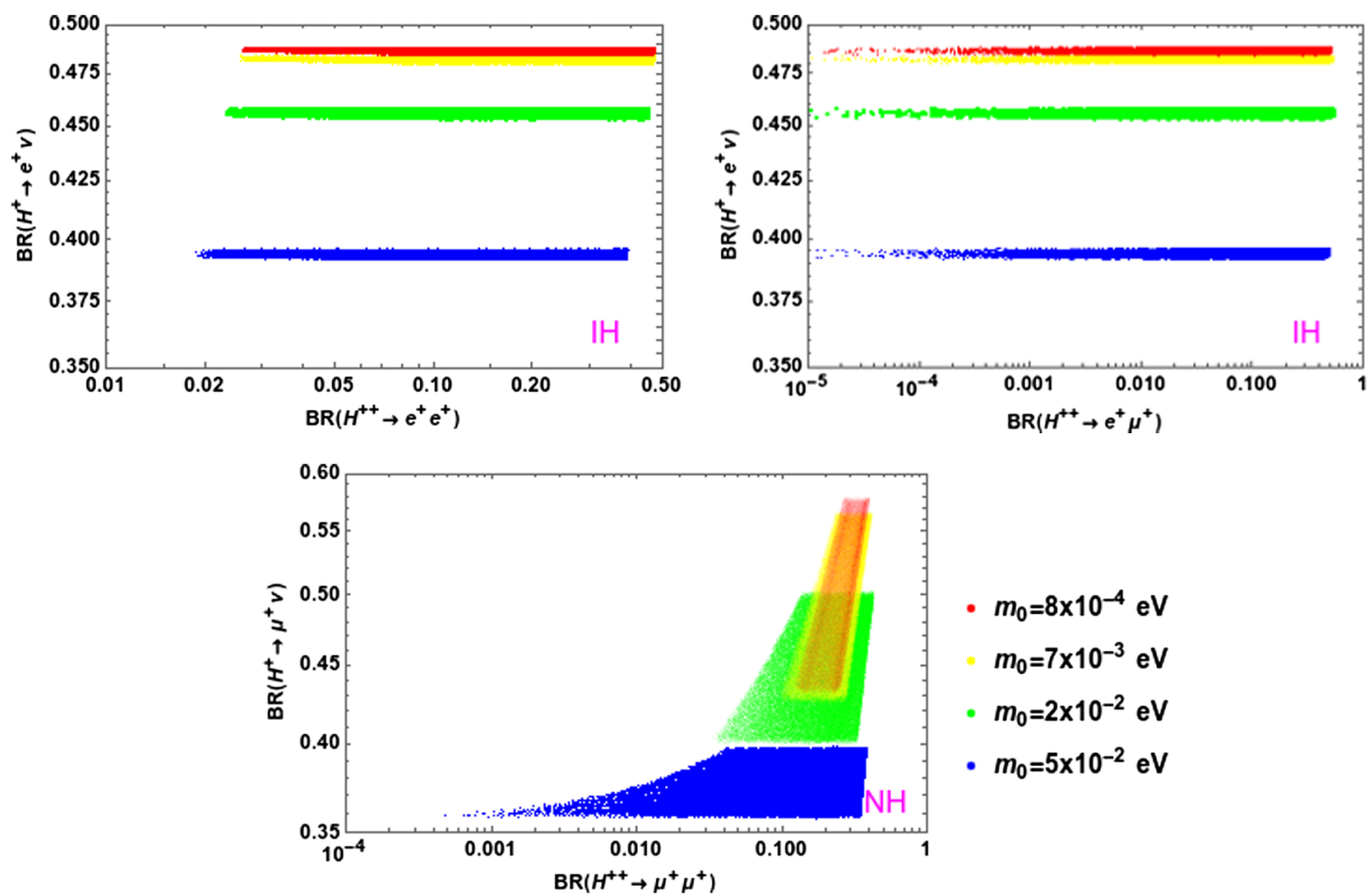

FIG. 3. Variation of leptonic branching ratios of singly charged Higgs with the leptonic branching ratios of doubly charged Higgs. Here $\nu$ implies all the neutrino states $\nu_{1,2,3}$. See the texts for the details.

As we quantify in the next section, the uncertainty in branching ratios can have a large impact on the theory cross section.

\section{PAIR-PRODUCTION CROSS SECTION FOR $\sqrt{s}=13$ TeV LHC}

In Fig. 4, we plot the production cross section of $H^{ \pm \pm}$as a function of $M_{H^{ \pm \pm}}$at LHC with $\sqrt{s}=13 \mathrm{TeV}$. We also show the cross section for a future $p p$ collider HE-LHC that can operate with center-of-mass energy $27 \mathrm{TeV}$. Here we show both pair $\left(p p \rightarrow H^{++} H^{--}\right)$and associated ( $p p \rightarrow H^{++} H^{-}+$H.c.) production modes. Cross sections for the production of $\mathrm{H}^{++} \mathrm{H}^{--}$(mediated by $\gamma^{\star} / Z^{\star}$ ) and $H^{++} H^{-}$(mediated by $W^{+\star}$ ) are comparable. As shown in Fig. 4, the production cross section of $H^{+} H^{--}$(mediated by $W^{-\star}$ ) is smaller than that of $\mathrm{H}^{++} \mathrm{H}^{-}$, which can be understood from parton distribution functions of the proton. At the $p \bar{p}$ collider, both are the same. We consider a $K$ factor as 1.25 [61] for the left panel of Fig. 4. In our analysis, we assume a degenerate mass spectrum for the singly and doubly charged Higgs.

The CMS and ATLAS Collaborations have already placed constraints on $M_{H^{ \pm \pm}}$by analyzing the leptonic decay channels of $H^{ \pm \pm}$[34,35]. A degenerate mass spectrum for charged scalars and $\operatorname{BR}\left(H^{ \pm \pm} \rightarrow l_{i}^{ \pm} l_{j}^{ \pm}\right)=100 \%$ have been
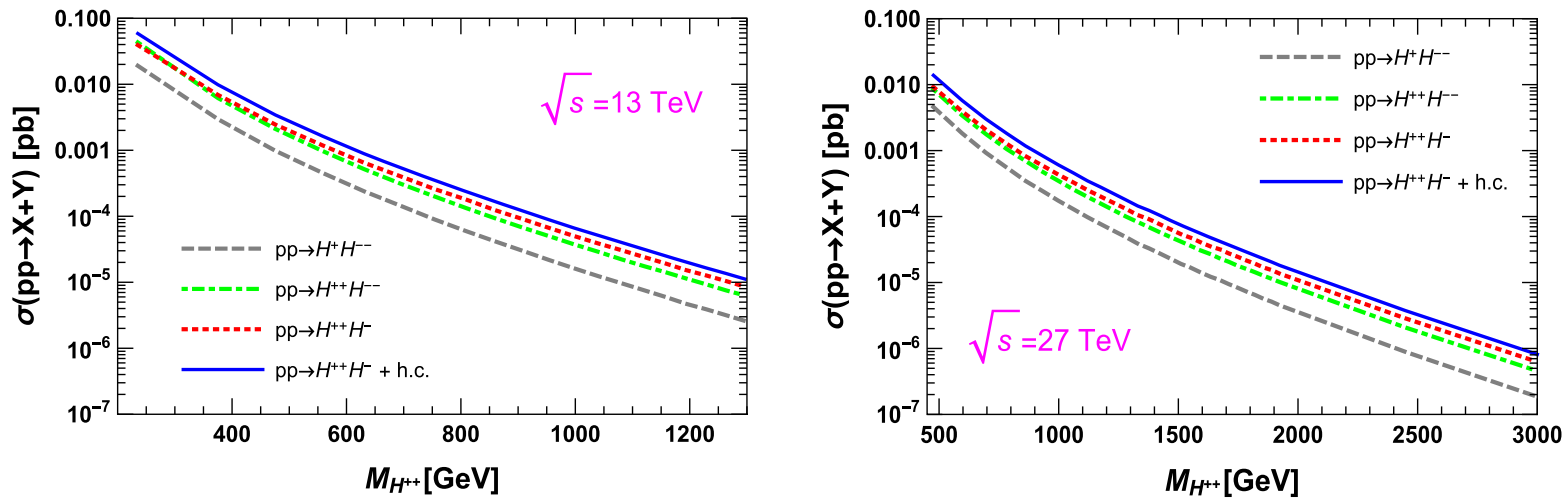

FIG. 4. Pair and associated production cross section of $H^{ \pm \pm}$as a function of $M_{H^{ \pm \pm}}$. 

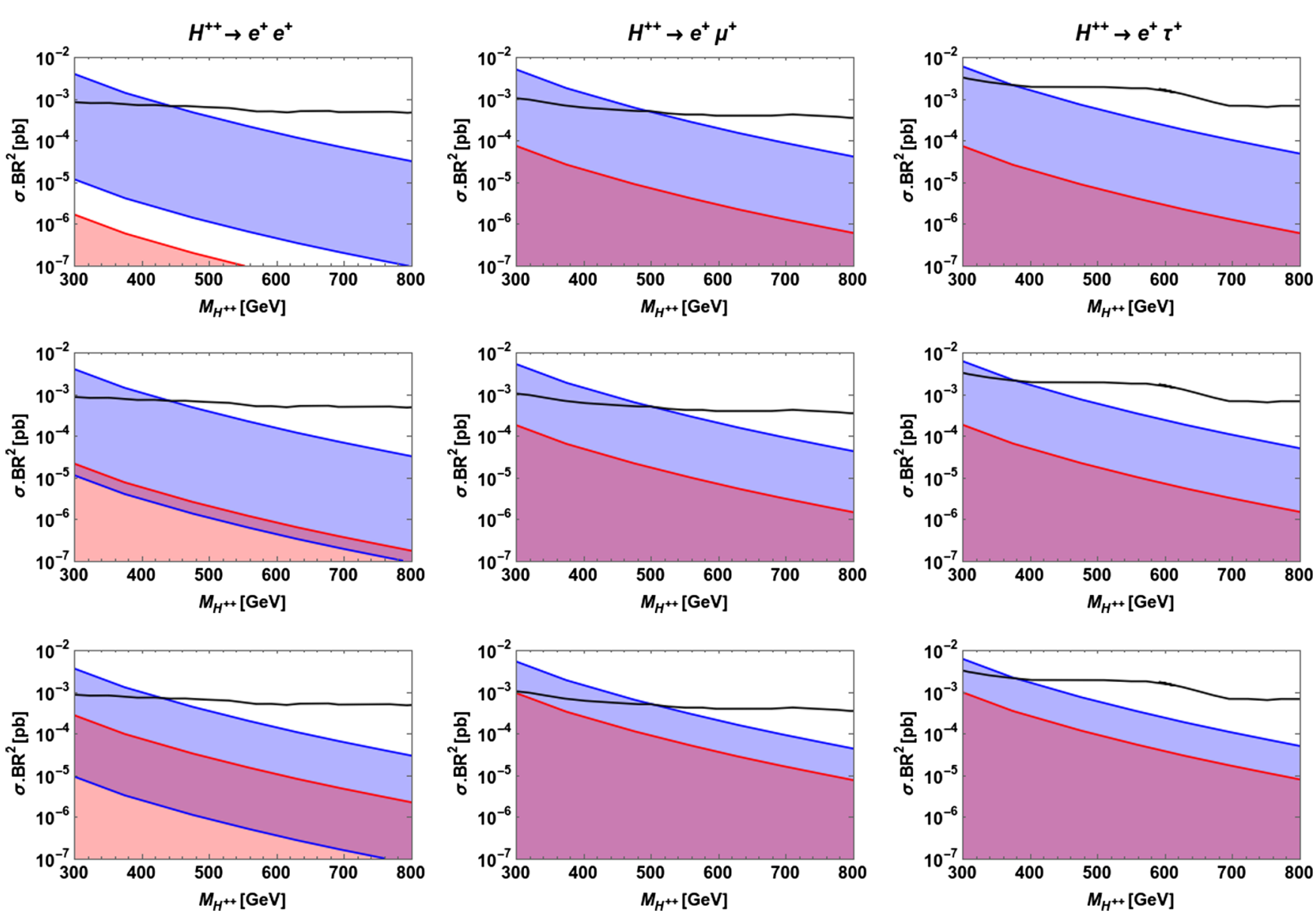

FIG. 5. The blue (red) bands for IH (NH) correspond to the theory cross sections for the channel $p p \rightarrow H^{++} H^{--} \rightarrow l_{i}^{+} l_{j}^{+} l_{k}^{-} l_{l}^{-}$obtained by including $3 \sigma$ variations of neutrino oscillation parameters. The black line represents the observed limit from CMS analysis [35]. The horizontal panels in rows $1-3$ represent $m_{0}=(0.0008,0.007,0.02) \mathrm{eV}$. In the first, second, and third columns we consider the decay of $H^{ \pm \pm}$to $e^{ \pm} e^{ \pm}, e^{ \pm} \mu^{ \pm}$, and $e^{ \pm} \tau^{ \pm}$, respectively.

assumed in the analysis. The CMS analysis focused on the trilepton and tetralepton final states originating from the leptonic decays of $H^{ \pm \pm}$and $H^{ \pm}$. ATLAS searches considered pair production of $H^{ \pm \pm}$and their subsequent decay into $e^{ \pm} e^{ \pm}, e^{ \pm} \mu^{ \pm}, \mu^{ \pm} \mu^{ \pm}$states. As a result of these searches, limits on $M_{H^{ \pm \pm}}$vary between 770 and $870 \mathrm{GeV}$ at a $95 \%$ C.L. The CMS Collaboration studied both pair and associated production channels of $H^{ \pm \pm}$and the subsequent decay of $H^{ \pm \pm}$ and $H^{ \pm}$to different leptonic states. Limits on $M_{H^{ \pm \pm}}$obtained from the combined study of both the channels vary between 535 and $820 \mathrm{GeV}$ at a $95 \%$ C.L., for $100 \%$ branching to each leptonic state. This limit varies between 396 and $712 \mathrm{GeV}$ if only the pair-production channel is considered. The most stringent constraint, $M_{H^{ \pm \pm}}>820 \mathrm{GeV}$, has been given by assuming $H^{ \pm \pm} \rightarrow e^{ \pm} \mu^{ \pm}$decay, and this takes into account both pair and associated productions. The CMS analysis [35] has further considered few benchmark points, and has given limits on $M_{H^{ \pm \pm}}$. However, the PMNS mixing angle $\theta_{13}$ has been assumed as zero, which is inconsistent with the present neutrino oscillation data. The above mentioned searches include pair and associated production of $H^{ \pm \pm}$and only its leptonic decay modes, so the observed limit on $M_{H^{ \pm \pm}}$is valid only for low triplet VEV $v_{\Delta} \leq 10^{-4} \mathrm{GeV}$, where the dileptonic branching is maximum. As this is evident from the discussion presented in the previous section, the maximum possible branching in each channel can never be $100 \%$; rather, it can be at most $73 \%$ (for $H^{ \pm \pm} \rightarrow \mu^{ \pm} \tau^{ \pm}$in $\mathrm{NH}$ ). Instead of considering $\operatorname{BR}\left(H^{ \pm \pm} \rightarrow l_{i}^{ \pm} l_{j}^{ \pm}\right)=100 \%$, we rescale the theory cross section with appropriate branching ratios. This somewhat weakens the individual bounds from different channels. In the context of the BNT model [62], it has been shown that by taking into account neutrino oscillation data, one can lower the current CMS bound on $M_{H^{ \pm \pm}}$. For illustration, we focus on the final states with $e^{ \pm} e^{ \pm} e^{\mp} e^{\mp}, e^{ \pm} \tau^{ \pm} e^{\mp} \tau^{\mp}$, and $e^{ \pm} \mu^{ \pm} e^{\mp} \mu^{\mp}$. Due to the absence of any cancellation region, the first channel is the least uncertain. We note that, apart from the dependency on neutrino oscillation parameters, the limit from an individual channel also depends on the value of lightest neutrino mass $m_{0}$. 
TABLE I. Maximum possible branching ratios for the decay mode $H^{ \pm \pm} \rightarrow l_{i}^{ \pm} l_{j}^{ \pm}$. We also show the corresponding lower limits on $M_{H^{ \pm \pm}}$in parentheses obtained from the channel $p p \rightarrow H^{++} H^{--} \rightarrow l_{i}^{+} l_{j}^{+} l_{i}^{-} l_{j}^{-}$(here $\left.l_{i}^{+}=e^{+} / \mu^{+} / \tau^{+}\right)$. We use Ref. [35] to derive the limits.

\begin{tabular}{|c|c|c|c|c|}
\hline \multicolumn{5}{|c|}{ Maximum value of $\mathrm{BR}\left(H^{ \pm \pm} \rightarrow l_{i}^{ \pm} l_{j}^{ \pm}\right)\left(M_{H^{ \pm \pm}}[\mathrm{GeV}]\right)$} \\
\hline Decay mode & $m_{0}=0.0008 \mathrm{eV}$ & $m_{0}=0.007 \mathrm{eV}$ & $m_{0}=0.02 \mathrm{eV}$ & Mass hierarchy \\
\hline$e^{ \pm} e^{ \pm}$ & 0.478 & 0.476 & $0.454(424)$ & $\mathrm{IH}$ \\
\hline$e^{ \pm} \mu^{ \pm}$ & $0.537(495)$ & $0.547(503)$ & $0.552(503)$ & $\mathrm{IH}$ \\
\hline$e^{ \pm} \tau^{ \pm}$ & $0.583(373)$ & $0.594(376)$ & $0.594(376)$ & $\mathrm{IH}$ \\
\hline$\mu^{ \pm} \mu^{ \pm}$ & $0.410(465)$ & $0.424(478)$ & $0.434(482)$ & $\mathrm{NH}$ \\
\hline$\mu^{ \pm} \tau^{ \pm}$ & $0.604(428)$ & $0.656(440)$ & $0.735(450)$ & $\mathrm{NH}$ \\
\hline$\tau^{ \pm} \tau^{ \pm}$ & $0.363(<200)$ & $0.382(<200)$ & $0.404(<200)$ & $\mathrm{NH}$ \\
\hline
\end{tabular}

In Fig. 5, we show the production cross sections of $p p \rightarrow H^{++} H^{--} \rightarrow e^{+} e^{+} e^{-} e^{-}, e^{+} \mu^{+} e^{-} \mu^{-}$, and $e^{+} \tau^{+} e^{-} \tau^{-}$ at the $\mathrm{LHC}$ for $\sqrt{s}=13 \mathrm{TeV}$. The colored band represents the variation of cross sections due to $3 \sigma$ uncertainty in neutrino oscillation parameters. As illustrative points, we choose three values of lightest neutrino mass, $m_{0}=0.0008$,
$0.007,0.02 \mathrm{eV}$, that fall in the hierarchical mass regime. The blue (red) band corresponds to the $\mathrm{IH}(\mathrm{NH})$ neutrino mass spectrum. The black line represents the observed limit from $13 \mathrm{TeV}$ CMS analysis [35]. For a given value of the lightest neutrino mass $m_{0}$, the upper boundary in these bands is determined from $\sigma\left(p p \rightarrow H^{++} H^{--}\right)$folded with
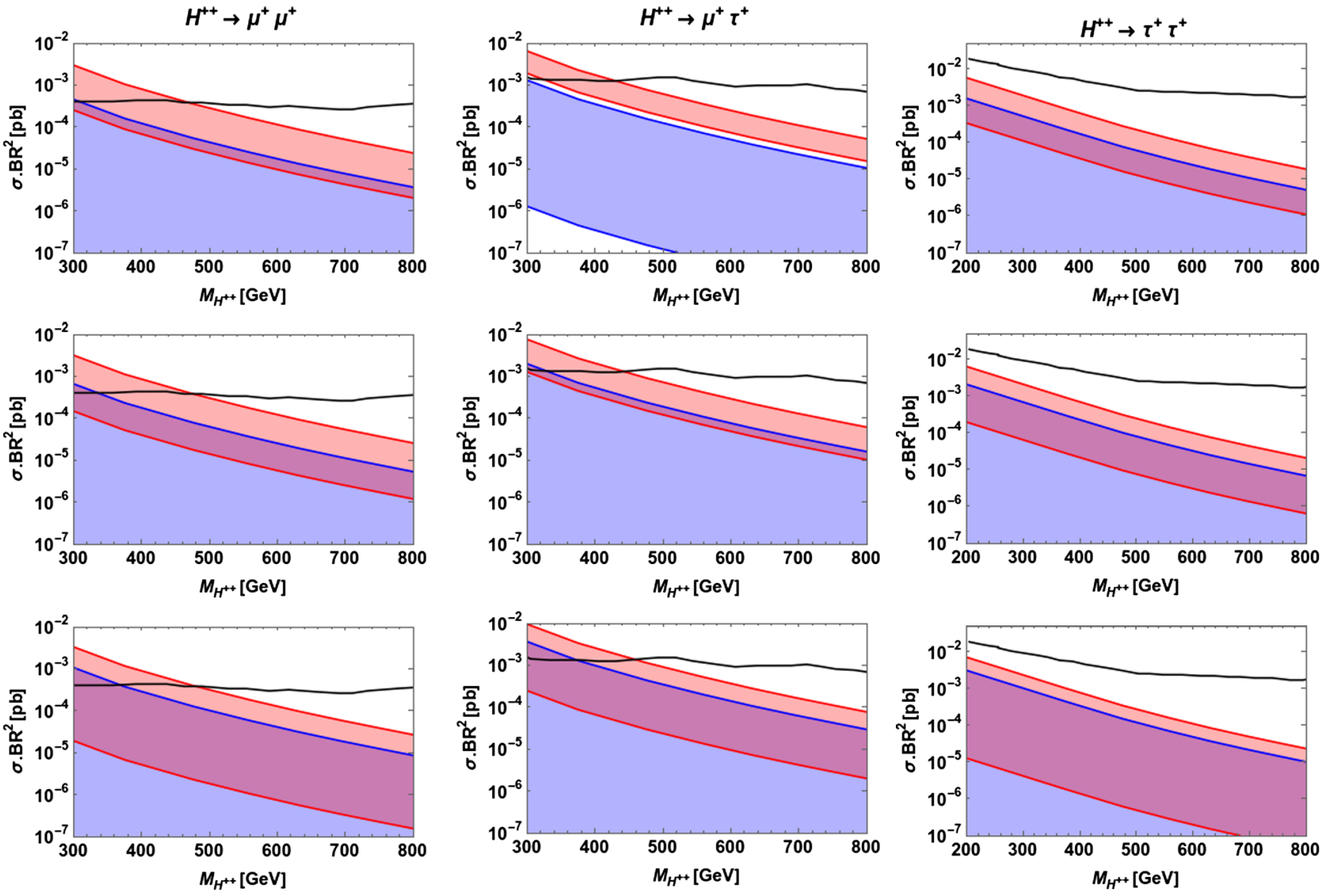

FIG. 6. The blue (red) bands for $\mathrm{IH}(\mathrm{NH})$ correspond to the theory cross sections for the channel $p p \rightarrow H^{++} H^{--} \rightarrow l_{i}^{+} l_{j}^{+} l_{k}^{-} l_{l}^{-}$obtained by including $3 \sigma$ variation of neutrino oscillation parameters. The black line represents the observed limit from CMS analysis [35]. The horizontal panels in rows $1-3$ represent $m_{0}=(0.0008,0.007,0.02) \mathrm{eV}$. In the first, second, and third columns we consider the decay of $H^{ \pm \pm}$to $\mu^{ \pm} \mu^{ \pm}, \mu^{ \pm} \tau^{ \pm}$, and $\tau^{ \pm} \tau^{ \pm}$, respectively. 
the square of maximum possible $\operatorname{BR}\left(H^{ \pm \pm} \rightarrow l_{i}^{ \pm} l_{j}^{ \pm}\right)$. Similarly, the lower line represents the minimum value of $\mathrm{BR}\left(H^{ \pm \pm} \rightarrow l_{i}^{ \pm} l_{j}^{ \pm}\right)$. A couple of points are in order:

(1) The total cross section has a large variation, specially for the $e^{+} \mu^{+} e^{-} \mu^{-}$and $e^{+} \tau^{+} e^{-} \tau^{-}$channels. The $e^{+} e^{+} e^{-} e^{-}$channel in $\mathrm{IH}$ is the least uncertain, as this has a definite lower value of the cross section.

(2) Due to a relatively smaller branching ratio, the cross sections in $\mathrm{NH}$ for these modes are lower than the maximal possible cross section in $\mathrm{IH}$.

(3) The drop in cross section for $e^{+} \mu^{+} e^{-} \mu^{-}$and $e^{+} \tau^{+} e^{-} \tau^{-}$occurs due to the cancellation between different terms in $M_{12}^{\nu}$ and $M_{13}^{\nu}$.

Taking into account the branching ratios, the limit from each of the leptonic channels somewhat weakens, as compared to the analysis presented in Ref. [35]. However, the combined limit might be comparable to that analysis. For the above modes, IH can give the best constraint. The cross section for $\mathrm{NH}$ is an order of magnitude smaller in the hierarchical limit, and therefore competitive limits cannot be placed on $M_{H^{ \pm \pm}}$in the above channels if light neutrinos follow $\mathrm{NH}$. We tabulate the predicted value of maximum possible branching ratios in Table I, where each entry represents the maximum possible value of $\operatorname{BR}\left(H^{ \pm} \rightarrow l_{i}^{ \pm} l_{j}^{ \pm}\right)$for a given value of $m_{0}$. The value within the bracket denotes the best lower limit on $M_{H^{ \pm \pm}}$, from each channel.

In Fig. 6, we present plots similar to that of Fig. 5, considering the decay of $H^{ \pm \pm}$to $\mu^{ \pm} \mu^{ \pm}, \mu^{ \pm} \tau^{ \pm}$, and $\tau^{ \pm} \tau^{ \pm}$. The different plots represent the production cross section of $p p \rightarrow H^{++} H^{--} \rightarrow \mu^{+} \mu^{+} \mu^{-} \mu^{-}, \mu^{+} \tau^{+} \mu^{-} \tau^{-}$, and $\tau^{+} \tau^{+} \tau^{-} \tau^{-}$ at the LHC for $\sqrt{s}=13 \mathrm{TeV}$. We use the same color code and the same values of lightest neutrino masses as used in Fig. 5. For these modes, the maximal possible cross sections in $\mathrm{NH}$ are higher as compared to IH. Therefore, competitive limits on $M_{H^{ \pm \pm}}$can be derived in the above mentioned channels, if NH is assumed. The derived limits on $M_{H^{ \pm \pm}}$are tabulated in Table I.

In the CMS search, combined limits have been presented which result from the combined analysis of both the pair and associated production channels. Also, for the benchmark studies, their analysis combines different leptonic modes. Such a study for the combined limit is beyond the scope of this paper.

As discussed in Refs. [63,64], the $3 \sigma$ sensitivity reach of a doubly charged Higgs in the minimal left-right symmetric model is less than $1.3 \mathrm{TeV}$, where c.m. energy $\sqrt{s}=$ $14 \mathrm{TeV}$ and $3 \mathrm{ab}^{-1}$ integrated luminosity have been assumed. Another study on charged Higgs in the context of the minimal supersymmetric model [65] compares the discovery potential of the HL-LHC and HE-LHC. In Ref. [66], the phenomenology of a $S U(2)$-singlet doubly charged scalar has been discussed at the HL-LHC. In Ref. [41], the pair production of $H^{ \pm \pm}$and its subsequent decay to same-sign dileptons has been explored at the $13 \mathrm{TeV}$
TABLE II. Branching ratio of $H^{ \pm \pm}$into different leptonic states in the case of $\mathrm{IH}$ for $m_{0}=0.007 \mathrm{eV}$.

\begin{tabular}{lllllll}
\hline \hline & $e^{ \pm} e^{ \pm}$ & $e^{ \pm} \mu^{ \pm}$ & $e^{ \pm} \tau^{ \pm}$ & $\mu^{ \pm} \tau^{ \pm}$ & $\mu^{ \pm} \mu^{ \pm}$ & $\tau^{ \pm} \tau^{ \pm}$ \\
\hline BR & 0.026 & 0.547 & 0.365 & 0.001 & 0.001 & 0.053 \\
\hline \hline
\end{tabular}

and $14 \mathrm{TeV}$ LHC for $M_{H^{ \pm \pm}}$up to $700 \mathrm{GeV}$. Figure 6 of Ref. [41] shows that the $13 \mathrm{TeV}$ and $14 \mathrm{TeV}$ cross sections are not very different. It is evident from Fig. 4 that the pairproduction cross section of $H^{ \pm \pm}$at the $13 \mathrm{TeV}$ LHC becomes smaller for higher masses. For $M_{H^{ \pm \pm}}=1.3 \mathrm{TeV}$, the cross section is less than $10^{-5} \mathrm{pb}$, whereas at the HE-LHC it is around $10^{-4} \mathrm{pb}$. Therefore, assuming $3 \mathrm{ab}^{-1}\left(15 \mathrm{ab}^{-1}\right)$ integrated luminosity, approximately 60 (3000) $H^{ \pm \pm}$with mass $1.3 \mathrm{TeV}$ can be produced at the HL-LHC (HE-LHC). An increase in sensitivity for higher mass ranges will be possible for the HE-LHC. Therefore, to study the discovery prospects of heavier $H^{ \pm \pm}$and $H^{ \pm}$, we consider the higher center-of-mass energy-i.e., the HE-LHC setup with $\sqrt{s}=27 \mathrm{TeV}$. $^{1}$

In the next section, we present the collider analysis for multilepton signatures of $H^{ \pm \pm}$, where we assume $\mathrm{BR}\left(H^{ \pm \pm} \rightarrow e^{ \pm} \mu^{ \pm}\right)=0.547$, corresponding to $m_{0}=$ $0.007 \mathrm{eV}$, and $\mathrm{IH}$ as neutrino mass ordering. The other branching ratios are given in Table II. For completeness in our analysis, we however consider all leptonic modes, with their corresponding branching ratios. Note that, other than the pair production by the Drell-Yan process, the photon fusion can also contribute to the pair production of doubly charged Higgs bosons. It has been pointed out in Ref. [52], that for $13 \mathrm{TeV}$, the channel contributes at most $10 \%$ to the pair production of doubly charged Higgs states. However, there are different issues, regarding large uncertainties in PDFs. Therefore, one needs to evaluate this channel carefully. We do not consider this channel in our present analysis.

\section{MULTILEPTON SIGNALS FROM $\boldsymbol{H}^{ \pm \pm}$AND $\boldsymbol{H}^{ \pm}$ FOR THE $\sqrt{s}=27$ TeV HE-LHC}

We consider the setup for a future $p p$ collider HE-LHC, that can operate with a c.m. energy $\sqrt{s}=27 \mathrm{TeV}$, and we analyze the trilepton and tetralepton channels in detail. To simulate the signal samples, we implement the model in FeynRules (v2.3) [68]. The UFO output is then fed into MadGraph5_aMC@NLO (v2.6) [69], which generates the parton-level events. We use the default PDFs NNPDF23LO1 [70] for computation. We perform parton showering and

\footnotetext{
${ }^{1}$ While preparing the manuscript for this work, Ref. [67] appeared in arXiv. It considers the pair production of doubly charged Higgs and subsequent decays at the HE-LHC. We consider both pair and associated production, oscillation parameter dependency, and the correct values of the leptonic branching ratios, which have largely been overlooked in the literature.
} 
hadronization with PYTHIA8 [71] and carry out the detector simulations with DELPHES (v3.4.1) [72]. Finally, data analysis and ploting is done in ROOT (v6.14/04) [73]. We choose the degenerate mass spectrum for charged Higgs states for which the most promising signals are tetralepton and trilepton final states, arising from the pair and associated production of doubly charged Higgs.

\section{A. $4 l$ final state}

This originates from the pair production of $\mathrm{H}^{++}$and its subsequent decays $H^{ \pm \pm} \rightarrow l^{ \pm} l^{ \pm}$. Therefore, the signal is represented by the following chain:

Signal: $p p \rightarrow H^{++} H^{--} \rightarrow l_{i}^{+} l_{j}^{+} l_{k}^{-} l_{l}^{-}$, (with $l_{i}^{ \pm}=e^{ \pm} / \mu^{ \pm} / \tau^{ \pm}$). The $\tau$ in the final state further decays into fully hadronic or leptonic final states. For our analysis, we consider leptonic decays of $\tau$, and therefore, we collect all the event samples with $e, \mu$ in the final state. There are a number of SM processes that can mimic the signal, and hence are considered as SM backgrounds. Here we list the following processes as the dominant SM backgrounds:

(1) $Z Z: p p \rightarrow Z Z \rightarrow l_{i}^{+} l_{i}^{-} l_{j}^{+} l_{j}^{-}$.

(2) $t \bar{t} Z: p p \rightarrow t \bar{t} Z \rightarrow l_{i}^{+} l_{j}^{-} l_{k}^{+} l_{k}^{-}+b \bar{b}+E_{T}$.

(3) $t \bar{t} W: p p \rightarrow t \bar{t} W^{ \pm} \rightarrow l_{i}^{+} l_{j}^{-} l_{k}^{ \pm}+b \bar{b}+E_{T}$.

(4) $t \bar{t}: p p \rightarrow t \bar{t} \rightarrow l_{i}^{+} l_{j}^{-}+b \bar{b}+E_{T}$.

(5) $V V V\left(V=Z\right.$ or $\left.W^{ \pm}\right): p p \rightarrow V V V, V \rightarrow l_{i}^{+} l_{i}^{-}$or $l_{i}^{ \pm} E_{T}$ or $j j$.

(6) $W Z: p p \rightarrow W^{ \pm} Z \rightarrow l_{i}^{ \pm} l_{j}^{+} l_{j}^{-}+E_{T}$.

(7) $t \bar{t} t \bar{t}: p p \rightarrow t \bar{t} t \bar{t} \rightarrow l_{i}^{+} l_{j}^{-} l_{k}^{+} l_{m}^{-}+2 b \bar{b}+E_{T}$.

Among all these, the $Z Z, t \bar{t} Z$, and $W^{ \pm} W^{\mp} Z$ processes lead to irreducible backgrounds. However, a few other SM processes, such as $t \bar{t}, t \bar{t} W$, and $W Z$ with their subsequent decays can also give rise to four-lepton final states, due to the misidentification of jets as leptons. Multilepton events $\left(N_{l}>4\right)$ from $p p \rightarrow Z Z Z \rightarrow 6 l(l=e, \mu)$ can also mimic the signal due to detector inefficiency in lepton reconstruction, or if the lepton is too soft, and does not pass the selection cuts. Additionally, one of the $Z$ bosons in the above mentioned background can decay to two hadronic taus, which can also mimic the signal. As we will show below, most of the backgrounds are reduced significantly after imposing a $Z$ veto, as well as selecting a window on the $l^{ \pm} l^{ \pm}$invariant mass.

In Fig. 4, we show the pair-production cross section of $H^{ \pm \pm}$for $\sqrt{s}=27 \mathrm{TeV}$. The cross section varies from $10^{-2} \mathrm{pb}$ for $M_{H^{ \pm \pm}}=400 \mathrm{GeV}$ to $10^{-6} \mathrm{pb}$ for $M_{H^{ \pm \pm}}=$ $3 \mathrm{TeV}$. As the cross section is gradually decreasing with increasing mass, it will be difficult to probe very heavy $H^{ \pm \pm}$. Here we present a benchmark point with $M_{H^{ \pm \pm}}=1 \mathrm{TeV}$ to show a detailed cut efficiency. We consider a triplet VEV $v_{\Delta}$ as $10^{-8} \mathrm{GeV}$. We reiterate that, for the analysis, we consider IH neutrino mass ordering and the following set of oscillation parameters, for which $H^{ \pm \pm} \rightarrow e^{ \pm} \mu^{ \pm}$is the most dominant decay channel with a branching ratio 0.547 :
(1) $\theta_{12}=0.6567, \theta_{13}=0.1567, \theta_{23}=0.7385$.

(2) $\phi_{1}=3.0614, \phi_{2}=5.9, \delta=0.2029$.

(3) $m_{1}=0.04902 \mathrm{eV}, m_{2}=0.04973 \mathrm{eV}$, and $m_{3}=$ $0.007 \mathrm{eV}$.

This set of parameters is assumed because it puts the strongest limit on $M_{H^{ \pm \pm}}$, as is evident from Table I. Another reason for selecting this particular set of parameters is to reduce the value of $\operatorname{BR}\left(H^{ \pm \pm} \rightarrow \tau^{ \pm} \tau^{ \pm}\right)$. The branching ratio of $H^{ \pm \pm}$decays to different leptonic flavor states for this set of parameters is shown in Table II. Note that the doubly charged Higgs predominantly decays to $e^{ \pm} \mu^{ \pm}$and $e^{ \pm} \tau^{ \pm}$final states.

We apply the following set of basic cuts on transverse momentum, pseudorapidity, and separation between two leptons: $P_{T}(l)>10 \mathrm{GeV}(l=e$ or $\mu),|\eta(l)|<2.5, \Delta R_{l l}>$ 0.4 , at the time of event generation in MadGraph5. For detector level analysis, the isolation condition for a lepton $(e, \mu)$ is defined as $\frac{\sum P_{T}(x)}{P_{T}(l)}<0.2$, where $\sum P_{T}(x)$ is the scalar sum of transverse momenta of all particles within a cone of radius $\Delta R<0.4$ around the lepton direction and $P_{T}(l)$ is the transverse momentum of the lepton. We assume a jet misidentification rate $^{2}$ of $10^{-3}$ [74].

In Fig. 7, we plot the distribution of different kinematical variables for both signal and SM backgrounds. The top-left plot in Fig. 7 shows the transverse momentum distribution of the leading lepton. It is evident from this figure that most of the background lies in the low- $P_{T}\left(l_{1}\right)$ region, and the signal peaks at high $P_{T}\left(l_{1}\right)$ for high $M_{H^{ \pm \pm}}$. The top-right plot in Fig. 7 shows the distribution of the same-sign dilepton invariant mass $M\left(l^{ \pm} l^{ \pm}\right)$. Here the signal distribution peaks at $M_{H^{ \pm \pm}}$, which is very clear and well separated from backgrounds. Such a distinguished peak of $M_{H^{ \pm \pm}}$at a high value of $M\left(l^{ \pm} l^{ \pm}\right)$distribution helps us to discover $H^{ \pm \pm}$. In the lower panel of Fig. 7, we show the distribution of opposite-sign dilepton invariant mass, which shows that most of the dominant backgrounds peak around the $Z$-boson mass. Therefore, a veto on opposite-sign dilepton invariant mass around $M_{Z}$ will reduce most of the backgrounds involving $Z$.

The above distributions motivate us to consider the following set of selection cuts that suppress backgrounds: A1: $N_{l}=4$. We demand four isolated leptons in the final state. The leptons are $e, \mu$.

A2: We demand that the sum of charges of the four leptons be zero.

A3: $\left|M\left(l^{+} l^{-}\right)-M_{Z}\right|>10 \mathrm{GeV}$. To remove the backgrounds including at least one $Z$ boson, we veto the lepton pairs with the same flavor but opposite charges inside the mass window $\left|M\left(l^{+} l^{-}\right)-M_{Z}\right|<10 \mathrm{GeV}$.

\footnotetext{
${ }^{2}$ The jet misidentification rate is defined as the rate by which jets are identified as leptons. There is a small chance for low- $P_{T}$ jets to be identified as leptons. Although it is small, a significant background cross section can result from misidentification because of the large production cross section of QCD jets at the LHC.
} 

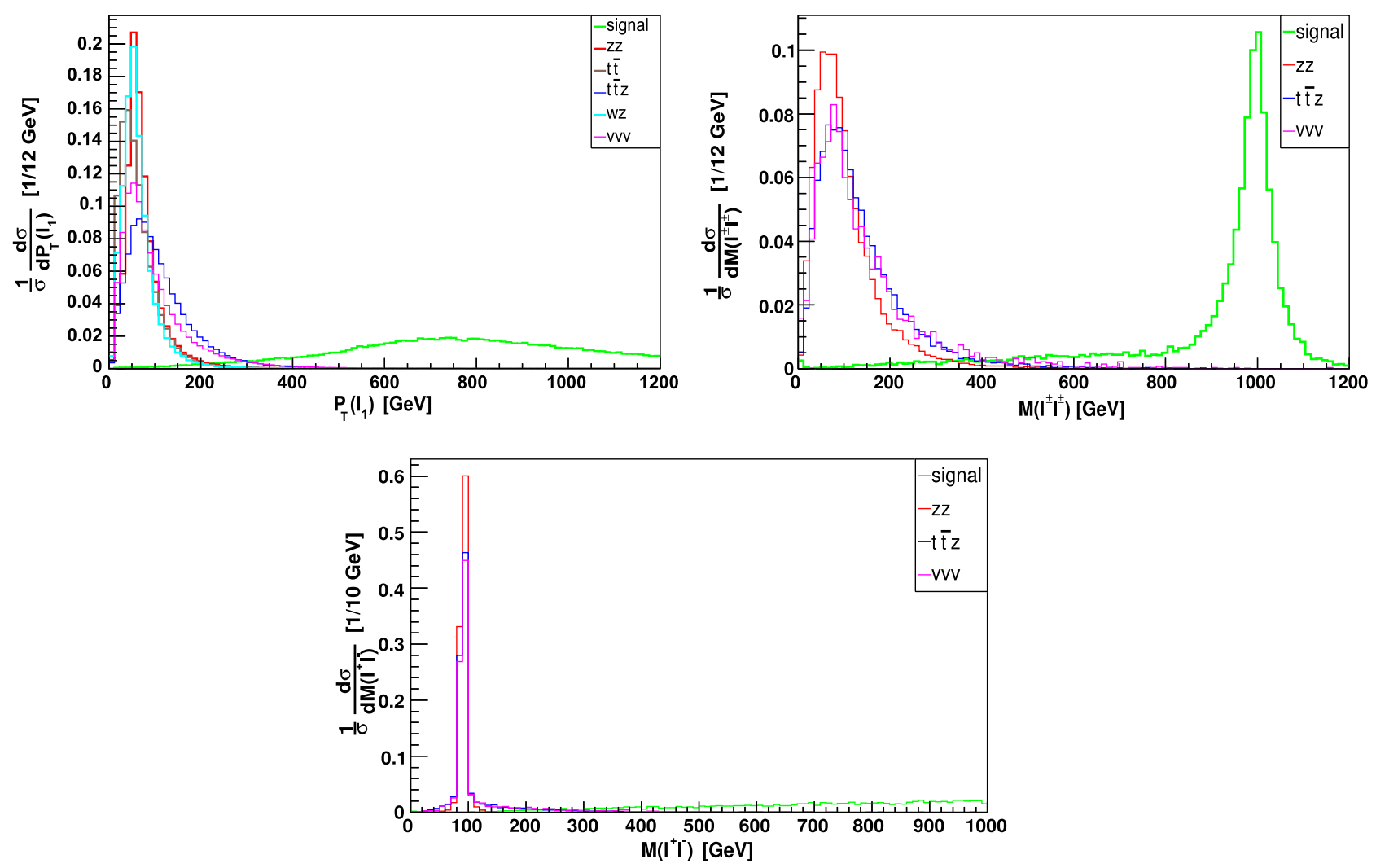

FIG. 7. Normalized distributions of transverse momentum of the leading lepton $P_{T}\left(l_{1}\right)$, the same-sign dilepton invariant mass $M\left(l^{ \pm} l^{ \pm}\right)$, and the opposite-sign dilepton invariant mass $M\left(l^{+} l^{-}\right)$for both $4 l$ signal and background events.

A4: $\left|M\left(l^{ \pm} l^{ \pm}\right)-M_{H^{ \pm \pm}}\right| \leq 50$. The signal events are selected by demanding a window on same-sign dilepton invariant mass within $M_{H^{ \pm \pm}} \pm 50 \mathrm{GeV}$.

In Table III, we show the changes in signal and background cross sections after each selection cut:

c1: $N_{l}=4$.

c2: $c_{1}$ and $\Sigma l_{\text {charge }}=0$.

c3: $c_{2}$ and $\left|M\left(l^{+} l^{-}\right)-M_{Z}\right|>10 \mathrm{GeV}$.

c4: $c_{3}$ and $\left|M\left(l^{ \pm} l^{ \pm}\right)-M_{H^{ \pm \pm}}\right| \leq 50$.

From Table III, the signal cross section before applying the cut is $0.2683 \mathrm{fb}$ for $M_{H^{ \pm \pm}}=1 \mathrm{TeV}$. Most of the dominant irreducible background appears from $Z Z$ with a cross section $83.559 \mathrm{fb}$. The channels $t \bar{t}$ and $W Z$ have huge cross sections compared to other backgrounds, but they result in a much lower number of four-lepton events. Demanding four leptons in the final state reduces the background cross section to a significant extent. An invariant mass window on same-sign dileptons finally helps to suppress almost all background events. It should be noted that we consider $t \bar{t}$ and $W Z$ backgrounds with 0 partons. In our analysis, the particles of interest are not jets but leptons, as the final state contains multileptons. We implemented stringent selection criteria on leptons. Hence, we expect that the involvement of multiparton processes will not substantially change the final results. However, we check the contribution of multiparton $t \bar{t}$ and $W Z$ processes, performing

TABLE III. Signal $\left(p p \rightarrow H^{++} H^{--} \rightarrow l_{i}^{+} l_{j}^{+} l_{k}^{-} l_{l}^{-}\right)$and background cross sections for $\sqrt{s}=27$ TeV after the different selection cuts for the channel. Here $l_{i}^{+}=e^{+} / \mu^{+}$.

\begin{tabular}{lcccccc}
\hline \hline & $\sigma[\mathrm{fb}]$ for signal & \multicolumn{4}{c}{$\sigma[\mathrm{fb}]$ for backgrounds } \\
\cline { 2 - 7 } & $M_{H^{ \pm \pm}}=1 \mathrm{TeV}$ & $Z Z$ & $\bar{t}$ & $t \bar{t} Z$ & $W Z$ & $V V V$ \\
\hline Before cut & 0.2683 & 83.559 & 142075 & 14.413 & 702.333 & 9.49 \\
After $c_{1}$ & 0.0597 & 18.56 & 9.9452 & 2.2616 & 0.9341 & 0.2668 \\
After $c_{2}$ & 0.0591 & 18.5035 & 9.9453 & 2.2368 & 0.48461 & 0.2568 \\
After $c_{3}$ & 0.0589 & 0.2031 & 7.1037 & 0.363 & 0.0913 & 0.0407 \\
After $c_{4}$ & 0.0194 & $\approx 0$ & $\approx 0$ & $\approx 0$ & $\approx 0$ & $\approx 0$ \\
\hline \hline
\end{tabular}


MLM matching in MadGraph5_aMC@NLO (v2.6) [69] and PYTHIA8 [71]. The result we obtain is similar to that in the case of 0-parton backgrounds.

Note that, although we present the $p p \rightarrow Z Z \rightarrow 4 l$ background in Table III, we also estimate $p p \rightarrow 4 l$, which includes the virtual photon contribution. The channel $p p \rightarrow l_{i}^{+} l_{j}^{+} l_{k}^{-} l_{l}^{-}$has the cross section $117.1 \mathrm{fb}$. We find a cross section $2.8 \mathrm{fb}$ after applying cut $c_{3}$. However, after cut $c_{4}$, the cross section becomes negligibly small. This is expected, as we choose a very large value of same-sign dilepton invariant mass, for which this background already falls off. We check the contribution of the $t \bar{t} t \bar{t}$ background, for which the cross section is $0.276 \mathrm{fb}$. After applying cuts $c_{2}$ and $c_{3}$, the cross sections are $0.027 \mathrm{fb}$ and $0.023 \mathrm{fb}$, respectively. After cut $c_{4}$, this cross section is reduced to an insignificant value. In addition, we also check the $t \bar{t} W$ background, which after cut $c_{4}$ gives an insignificant contribution. Although the SM background cross section is much higher than that of the signal before applying cut $c_{1}$, the backgrounds become insignificant after applying selection cuts. We find that, with $1000 \mathrm{fb}^{-1}$ luminosity, 19 events can be obtained for $M_{H^{ \pm \pm}}=1 \mathrm{TeV}$. We give the variation of the number of events versus the mass of the doubly charged Higgs in Fig. 9.

\section{B. $3 l$ final state}

Here we consider the signal containing trileptons (two same-sign leptons and another of opposite sign) and missing transverse energy $E_{T}$ in the final state. Associated production of $H^{ \pm \pm}$with $H^{ \pm}$and their subsequent leptonic decay dominantly contribute to the desired signal events. However, pair production of $H^{ \pm \pm}$also contributes to the same when at least one hadronically decaying tau lepton is present in the decay products of $H^{ \pm \pm}$. Therefore, the signal events we are analyzing originate from the following decay chains:

PP: $p p \rightarrow H^{++} H^{--} \rightarrow l_{i}^{+} l_{j}^{+} l_{k}^{-} l_{l}^{-}$(where $l_{i}^{+}=e^{+} / \mu^{+} / \tau^{+}$). AP: $p p \rightarrow H^{++} H^{-}+$H.c. $\rightarrow l_{i}^{+} l_{j}^{+} l_{k}^{-} \nu \quad$ (where $\quad l_{i}^{+}=$ $\left.e^{+} / \mu^{+} / \tau^{+}\right)$

We consider the following dominant SM backgrounds:

(1) $W Z: p p \rightarrow W^{ \pm} Z \rightarrow l_{i}^{ \pm} l_{j}^{+} l_{j}^{-}+E_{T}$.

(2) $Z Z: p p \rightarrow Z Z \rightarrow l_{i}^{+} l_{i}^{-} l_{j}^{+} l_{j}^{-}$.

(3) $V V V\left(V=Z\right.$ or $\left.W^{ \pm}\right): p p \rightarrow V V V, V \rightarrow l_{i}^{+} l_{i}^{-} / l_{i}^{ \pm} E_{T}^{\dagger} / j j$.

(4) $t \bar{t} W: p p \rightarrow t \bar{t} W^{ \pm} \rightarrow l_{i}^{+} l_{j}^{-} l_{k}^{ \pm}+b \bar{b}+E_{T}$.

(5) $D Y: p p \rightarrow Z / \gamma \rightarrow l_{i}^{+} l_{i}^{-}$.

(6) $t \bar{t}: p p \rightarrow t \bar{t} \rightarrow l_{i}^{+} l_{j}^{-}+b \bar{b}+E_{T}$.

(7) $t \bar{t} Z: p p \rightarrow t \bar{t} Z \rightarrow l_{i}^{+} l_{i}^{-} l_{j}^{+} l_{j}^{-}+b \bar{b}+E_{T}^{\prime}$.

The $W Z$ and $V V V$ channels result in irreducible background events with comparatively higher $E_{T}$. $Z Z$ and $t \bar{t} Z$ also contribute to the background when one among the leptons in the final state is a hadronic tau or is left undetected. The Drell-Yan $(D Y)$, and $t \bar{t}$ processes give trilepton events when a jet fakes as a lepton.
To simulate the signal and backgrounds, we generate events in MadGraph5, where we are applying the basic cuts $P_{T}(l)>10 \mathrm{GeV},|\eta(l)|<2.5, \Delta R_{l l}>0.4$. Here we consider the same neutrino mass pattern and oscillation parameters that we have considered for $4 l$ signal analysis in the previous subsection. For this set of parameters, $H^{ \pm}$ branching ratios are $e^{ \pm} \nu: \mu^{ \pm} \nu: \tau^{ \pm} \nu=0.48: 0.28: 0.24$. As the branching into muon and tau are very similar, we consider all the leptonic states in our analysis. We consider the mass of charged Higgs as $1 \mathrm{TeV}$ and show the distribution of transverse momentum of the leading lepton $P_{T}\left(l_{1}\right)$, the missing transverse energy $\mathscr{E}_{T}^{\prime}$, the opposite-sign dilepton invariant mass $M\left(l^{+} l^{-}\right)$, and the same-sign dilepton invariant mass $M\left(l^{ \pm} l^{ \pm}\right)$in Fig. 8. The two contributions to the signal events-i.e., AP and PP-are plotted separately. Most of the background events are distributed in the region of $P_{T}\left(l_{1}\right) \leq 200 \mathrm{GeV}$, as the leptons in the background originate from SM particles, but not from a heavy resonance. The signal sample, on the contrary, shows a peak at $P_{T}\left(l_{1}\right)>200 \mathrm{GeV}$. A comparison in the $E_{T}$ distribution between signal and background shows that most of the background events contain $E_{T}<200 \mathrm{GeV}$. Signal events coming from the AP channel contain more $E_{T}$ compared to those of the PP channel. This occurs because in the AP channel, $E_{T}^{\prime}$ results from the direct decay of $H^{ \pm}$into a lepton and neutrino. For the PP channel, the source of $E_{T}$ is the decay of $\tau$ into semileptonic/leptonic final states, or the mismeasurement of jet energy. We also show other distributions, such as opposite-sign and samesign dilepton invariant mass distributions. In the oppositesign dilepton invariant mass distribution, background events peak around $Z$ mass. In the same-sign dilepton invariant mass distribution, signal events peak at a higher value of $M\left(l^{ \pm} l^{ \pm}\right)$, as they directly originate from $H^{ \pm \pm}$. Most of the background events are accumulated in the lower- $M\left(l^{ \pm} l^{ \pm}\right)$region.

To suppress the backgrounds, we consider the following selection criteria:

A1: Number of leptons, $N_{l}=3$. We demand exactly three isolated leptons in the final state.

A2: Sum of the lepton charge is $\pm 1,\left|\Sigma l_{\text {charge }}\right|=1$. The charge configurations of leptons are either ++- or --+ .

A3: Invariant mass of opposite-sign leptons falls within a $10 \mathrm{GeV}$ mass window around $M_{Z},\left|M\left(l^{+} l^{-}\right)-M_{Z}\right|>$ $10 \mathrm{GeV}$. This cut effectively removes most of the backgrounds from $Z$ decay.

A4: The transverse momentum of the leading lepton, $P_{T}\left(l_{1}\right) \geq 150 \mathrm{GeV}$. We implement a $150 \mathrm{GeV}$ cut on $P_{T}$ of the leading lepton, as SM background events contain soft leptons compared to those of signal events.

A5: The missing transverse energy, $E_{T}>100 \mathrm{GeV}$. We collect events with $E_{T}>120 \mathrm{GeV}$. 

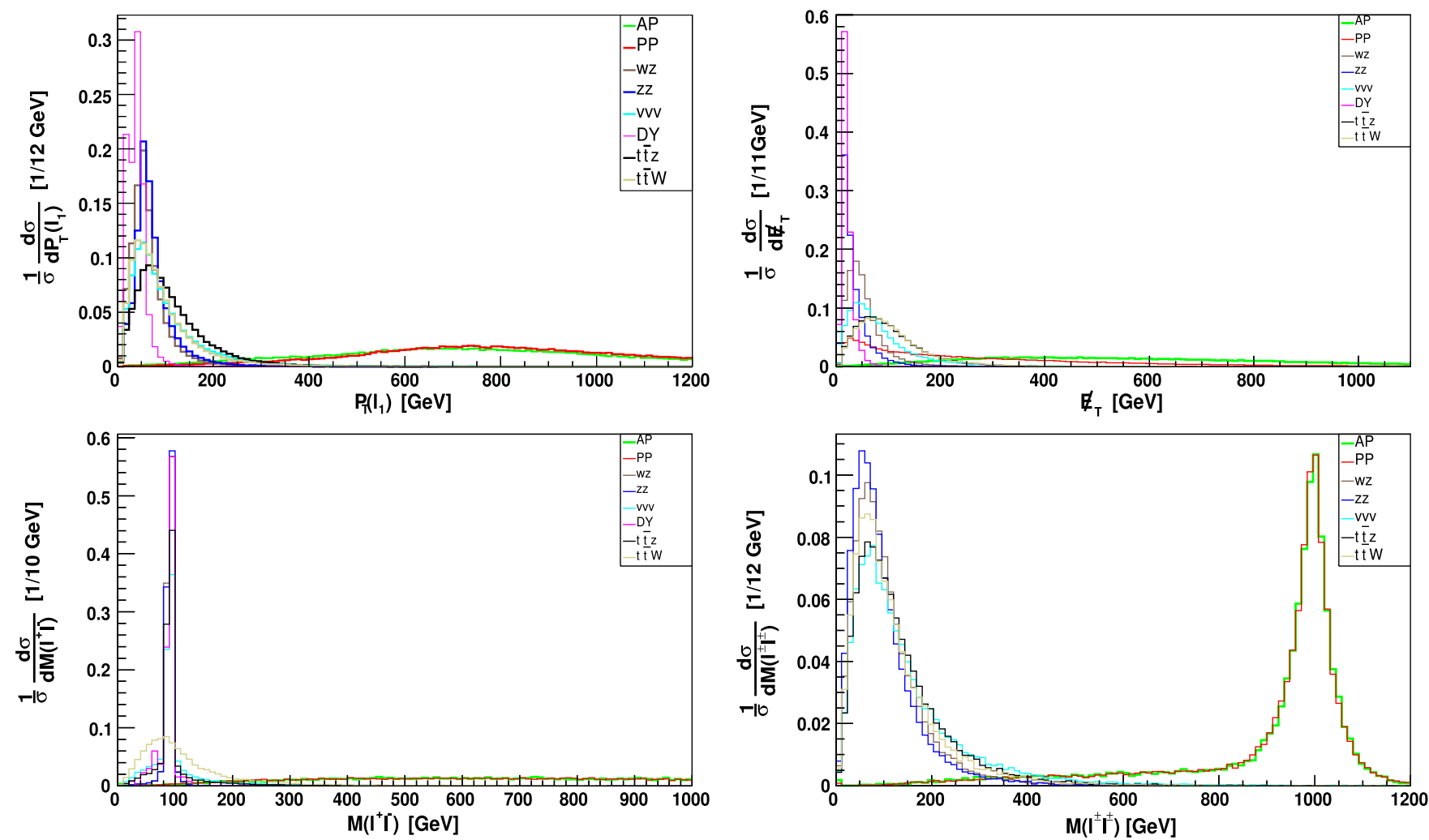

FIG. 8. Normalized distribution of the transverse momentum of the leading lepton $P_{T}\left(l_{1}\right)$, the missing transverse energy $\mathscr{E}_{T}$, the opposite-sign dilepton invariant mass $M\left(l^{+} l^{-}\right)$, and the same-sign dilepton invariant mass $M\left(l^{ \pm} l^{ \pm}\right)$for both $3 l$ signal and background events.

A6: Same-sign dilepton invariant mass, $\mid M\left(l^{ \pm} l^{ \pm}\right)-$ $M_{H^{ \pm \pm}} \mid \leq 50$; i.e., events within $100 \mathrm{GeV}$ are selected.

In Table IV, we show the signal and background cross sections after applying each of the selection cuts:

c1: $N_{l}=3$.

c2: $c_{1}$ and $\left|\Sigma l_{\text {charge }}\right|=1$.

c3: $c_{2}$ and $\left|M\left(l^{+} l^{-}\right)-M_{Z}\right|>10 \mathrm{GeV}$.

c4: $c_{3}$ and $P_{T}\left(l_{1}\right) \geq 150 \mathrm{GeV}$.

c5: $c_{4}$ and $E_{T} \geq 120 \mathrm{GeV}$.

c6: $c_{5}$ and $\left|M\left(l^{ \pm} l^{ \pm}\right)-M_{H^{ \pm \pm}}\right| \leq 50 \mathrm{GeV}$.
The partonic cross section for the AP channel is $0.4501 \mathrm{fb}$ before applying any cut. For the PP channel, it is $0.2683 \mathrm{fb}$. Most of the backgrounds have very large cross sections compared to the signal. At the detector level, demanding three leptons reduces the cross sections significantly. The $Z$ veto (cut $c_{3}$ ) and demanding a high $P_{T}$ of the leading lepton (cut $c_{4}$ ) removes many backgrounds. The missing transverse energy cut $\left(c_{5}\right)$ also helps to suppress backgrounds. SM processes like the Drell-Yan $(D Y)$ and $t \bar{t}$, which give $3 l$ due to jets faking as leptons, are left with negligible cross sections after applying the above

TABLE IV. Backgrounds and signal cross sections for $\sqrt{s}=27 \mathrm{TeV}$ after the final selection cuts for the $3 l$ final state. For signal $M_{H^{ \pm \pm}}=1 \mathrm{TeV}$.

\begin{tabular}{|c|c|c|c|c|c|c|c|}
\hline & \multicolumn{2}{|c|}{$\sigma(\mathrm{fb})$ for signal } & \multicolumn{5}{|c|}{$\sigma(\mathrm{fb})$ for backgrounds } \\
\hline & AP & PP & $W Z$ & $Z Z$ & $V V V$ & $t \bar{t} W$ & $t \bar{t} Z$ \\
\hline Before cut & 0.4501 & 0.2683 & 702.333 & 83.5597 & 9.49 & 20.38 & 14.4134 \\
\hline After $c_{1}$ & 0.1456 & 0.1052 & 195.368 & 22.104 & 2.48 & 3.868 & 4.637 \\
\hline After $c_{2}$ & 0.1453 & 0.1051 & 195.2 & 22.09 & 2.476 & 3.853 & 4.629 \\
\hline After $c_{3}$ & 0.14497 & 0.1048 & 17.158 & 1.8943 & 1.174 & 3.391 & 1.577 \\
\hline After $c_{4}$ & 0.14493 & 0.1047 & 0.899 & 0.122 & 0.265 & 0.725 & 0.354 \\
\hline After $c_{5}$ & 0.14074 & 0.0672 & 0.3582 & 0.0192 & 0.1138 & 0.3053 & 0.1503 \\
\hline After $c_{6}$ & 0.0793 & 0.0308 & $\approx 0$ & $\approx 0$ & 0.0005 & 0.001 & $\approx 0$ \\
\hline
\end{tabular}



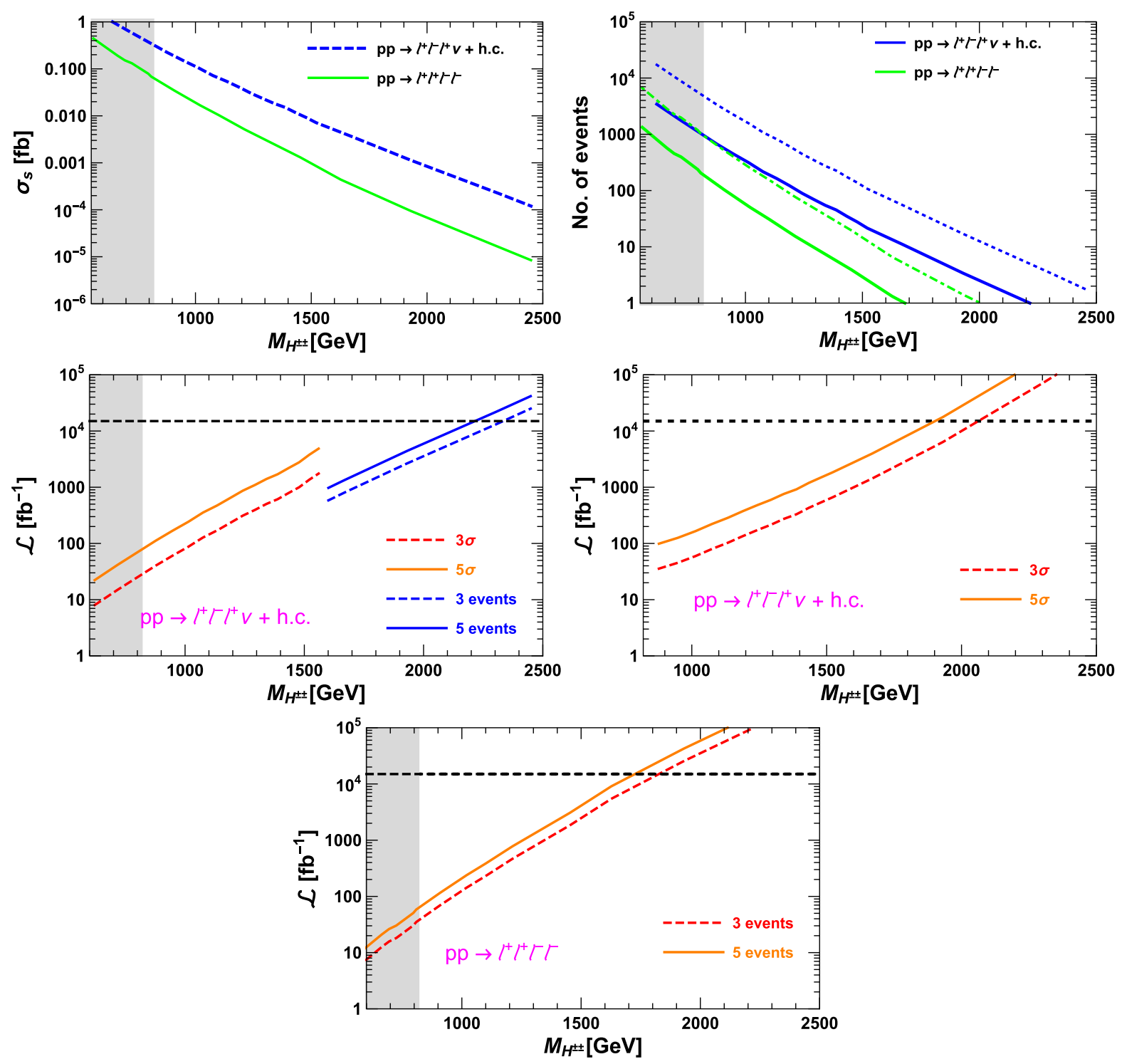

FIG. 9. Upper panels: Variation of trilepton and tetralepton cross sections after all cuts (left plot) and number of events (right plot) for $3 \mathrm{ab}^{-1}$ (solid line) and $15 \mathrm{ab}^{-1}$ (dashed, dot-dashed lines) luminosity as a function of $M_{H^{ \pm \pm}}$. Middle panels: Variation of required luminosity to reach $3 \sigma$ and $5 \sigma$ significance, and number of events $N=3,5$ vs $M_{H^{ \pm \pm}}$for a trilepton signal. Lower panel: Variation of required luminosity to observe a number of events $N=3,5$ vs $M_{H^{ \pm \pm}}$for a tetralepton signal. The gray shaded band represents the excluded region from the CMS search [35].

mentioned cuts. Therefore, we do not show them explicitly in Table IV. Similarly, the virtual photon contribution to the $3 l$ signal does not survive at the end. Cut $c_{5}$ reduces the AP and PP cross sections to one sixth and one ninth of their initial values, respectively. Although at the partonic level, signal cross sections are very small compared to those of SM backgrounds, we suitably choose selection criteria that suppress most of the backgrounds and keep a significant number of signal events. The SM background cross section is finally reduced to around $0.0015 \mathrm{fb}$. For an integrated luminosity of $1000 \mathrm{fb}^{-1}$, we get around 79 and 30 signal events for the AP and PP channels, respectively. Note that most of the backgrounds in both the trilepton and tetralepton channels drop off after including the invariant mass cuts. For completeness, however, we consider all the backgrounds and show the effects of selection cuts.

We show the variation of effective cross sections of the $4 l$ and $3 l$ final states with $M_{H^{ \pm \pm}}$in Fig. 9. The green line in the top-left plot represents the cross section for the $4 l$ final state. The blue dashed line denotes the cross section for the $3 l$ final state, taking into account both pair and associated production. Although the dominant contribution for the $3 l$ final state comes from associated production of $H^{ \pm \pm}$, the pair-production channel also contributes a significant amount. In the topright plot, we show the variation of the number of events as a 
function of $M_{H^{ \pm \pm}}$. We consider two different luminosities: $3 \mathrm{ab}^{-1}$, and the projected $15 \mathrm{ab}^{-1}$ of HE-LHC.

Finally, we calculate the statistical significance for the trilepton channel:

$$
\mathcal{S}=\frac{s}{\sqrt{s+b}}
$$

In the above, $s$ and $b$ are the numbers of signal and background events after all of the above mentioned selection cuts. The required luminosity $(\mathcal{L})$ to achieve a desired significance $(\mathcal{S})$ therefore scales as

$$
\sqrt{\mathcal{L}}=\mathcal{S} \frac{\sqrt{\sigma_{b}+\sigma_{s}}}{\sigma_{s}}
$$

where $\sigma_{s}$ and $\sigma_{b}$ are the signal and background cross sections after all the cuts. We obtain $3.3 \sigma$ significance for a $1 \mathrm{TeV}$ doubly charged Higgs with $100 \mathrm{fb}^{-1}$ luminosity, in the trilepton channel. After including systematic uncertainty, ${ }^{3}$ the significance decreases to $3.2 \sigma$. For the tetralepton final states, and for the trilepton channel in the higher-mass range, there is no SM background. This happens due to the very high invariant mass cut of the same-sign dilepton. Therefore, for these cases, we simply scale the required luminosity as

$$
\mathcal{L}=\frac{N}{\sigma_{s}},
$$

where $N$ is the number of signal events, and $\sigma_{s}$ is the cross section after all cuts. The two plots in the middle panels of Fig. 9 show the required luminosity to observe trilepton states for the $M_{H^{ \pm \pm}}$between 820 and $2500 \mathrm{GeV}$. In the left plot, we impose a flat $100 \mathrm{GeV}$ window of invariant mass $l^{ \pm} l^{ \pm}$around $M_{H^{ \pm \pm}}$. The orange and red lines have been obtained by using Eq. (26), where the background is sizeable. In the higher-mass range, there is almost no background. We therefore use Eq. (27). $H^{ \pm \pm}$with a mass $\sim 2.2 \mathrm{TeV}$ can be discovered with five events. We also present a conservative estimate in the right plot of the middle panels, where we impose a cut on the same-sign dilepton invariant mass $M\left(l^{ \pm} l^{ \pm}\right)>820 \mathrm{GeV}$, for which we have a constant background, $0.008 \mathrm{fb}$. This shows that $\sim 2 \mathrm{TeV} M_{H^{ \pm \pm}}$can be discovered in the trilepton channel with an integrated luminosity of $15 \mathrm{ab}^{-1}$. For the tetralepton final state (lower panel of Fig. 9), we find that approximately five events can be observed for $M_{H^{ \pm \pm}}=$ $1.7 \mathrm{TeV}$ with the same luminosity.

The analysis that we present here depends on the chosen final state and the branching ratios, as well as the selection

\footnotetext{
${ }^{3}$ We consider $2 \%-6 \%$ uncertainty for the lepton identification [35] and 5\% uncertainty on the missing transverse enery scale [75].
}

cuts. Additionally, the results presented are at the leadingorder (LO) level. However, we would like to present an estimate of the results taking into account the $K$ factor for leading background. For signal, the $K$ factors at the $14 \mathrm{TeV}$ and $100 \mathrm{TeV}$ LHC have been calculated in Ref. [76], which are not widely different. For background cross sections, we calculate the next-to-leading-order (NLO) corrections at the $27 \mathrm{TeV}$ LHC using MADGRAPH5_AMC@NLO (v2.6) [69]. The $K$ factor for $t \bar{t} W(V V V)$ is 1.86 (2.2). Considering the $\mathrm{LO}$ cross sections, the statistical significance $(\mathcal{S})$ for the $3 l$ signal (with $M_{H^{ \pm \pm}}=1 \mathrm{TeV}$ and $\mathcal{L}=100 \mathrm{fb}^{-1}$ ) is 3.29. If we include $K$ factors, the significance will increase to 3.6. Including NLO corrections to the pair production of doubly charged Higgs $(K=1.25)$, the discovery reach of $H^{ \pm \pm}$in the tetralepton final state can extend up to $1.8 \mathrm{TeV}$. We also cross-check the discovery potential of a very heavy $H^{ \pm \pm}$at the HE-LHC. We would like to point out that for a very large mass, such as $M_{H^{ \pm \pm}}=4 \mathrm{TeV}$, the pair-production cross section via the $D Y$ process drops to $\sigma \sim 3 \times 10^{-5} \mathrm{fb}$. Folded with approximately $50 \%$ branching ratios (note that the branching ratio in any of the leptonic final states cannot be $100 \%$ ), the final cross section for the four-lepton channel becomes very small. Due to this, we do not obtain any signal event with $15 \mathrm{ab}^{-1}$ luminosity for $4 \mathrm{TeV}$ doubly charged Higgs mass.

\section{CONCLUSION}

We analyze the discovery prospects of the doubly charged Higgs - a particle content of the type-II seesaw model in $p p$ colliders (HE-LHC). We focus on the region of small triplet VEV, where the doubly and singly charged Higgs naturally decay to same-sign dileptons and lepton plus neutrino, respectively. We analyze in detail multilepton signatures containing trilepton and tetralepton final states. The model signatures in this low-VEV regime strongly depend on the neutrino oscillation parameters, the neutrino mass hierarchy, and the lightest neutrino mass scale. We perform a robust estimation of the maximal possible branching ratios that each of the leptonic modes can accommodate. The constraint on doubly charged Higgs mass from individual leptonic channels somewhat weakens after taking into account correct branching ratios. The doubly charged Higgs and singly charged Higgs couple to the leptons through the same Yukawa coupling. We explore the relation between the branching ratios of singly and doubly charged Higgs decays. Our major findings are

(1) The branching of doubly charged Higgs into samesign leptons is augmented with large variation due to the uncertainty of neutrino oscillation parameters. We find that in the IH neutrino mass spectrum, and among all the leptonic decay modes of $H^{ \pm \pm}$, the $e^{ \pm} e^{ \pm}$mode is the least uncertain for the entire range of lightest neutrino mass $m_{0}$. This decay mode predicts a lower value of branching ratio, which is $\operatorname{BR}\left(H^{ \pm \pm} \rightarrow e^{ \pm} e^{ \pm}\right)>0.015$. Therefore, the 
observation of doubly charged Higgs in any other leptonic decay mode except $e^{ \pm} e^{ \pm}$with an upper limit on the branching ratio $\mathrm{BR}\left(H^{ \pm \pm} \rightarrow e^{ \pm} e^{ \pm}\right)<$ 0.015 will rule out $\mathrm{IH}$. The singly charged Higgs decay to $H^{ \pm} \rightarrow e^{ \pm} \nu$ is the least uncertain among all charged Higgs decays, with a predicted branching ratio that varies within $\sim 33 \%-50 \%$ for the variation of the lightest neutrino mass $m_{0} \sim 10^{-4} \mathrm{eV}-1 \mathrm{eV}$.

(2) The interaction of $H^{ \pm \pm}$and $H^{ \pm}$with leptons is governed by the same Yukawa couplings, and hence their leptonic branching ratios are related. In $\mathrm{IH}$, for a fixed $m_{0}$, and for a fixed value of $\operatorname{BR}\left(H^{ \pm \pm} \rightarrow e^{ \pm} e^{ \pm}\right)$, there is a small variation in $\mathrm{BR}\left(H^{ \pm} \rightarrow e^{ \pm} \nu\right)$. A similar result also exists between $\operatorname{BR}\left(H^{ \pm \pm} \rightarrow e^{ \pm} \mu^{ \pm}\right)$and $\operatorname{BR}\left(H^{ \pm} \rightarrow e^{ \pm} \nu\right)$.

(3) We perform a detailed analysis to find out the discovery prospect of trilepton and tetralepton final states at a future $p p$ collider that can operate with a c.m. energy $\sqrt{s}=27 \mathrm{TeV}$. We consider both the pair and associated productions, and a benchmark point of $H^{ \pm \pm}$with a mass $1 \mathrm{TeV}$. In the tetralepton final state, we find that using $1000 \mathrm{fb}^{-1}$ integrated luminosity, 19 events can be observed. In the trilepton final state, we find that the same mass can be discovered at the $27 \mathrm{TeV}$ LHC with a significance of more than $5 \sigma$ for $1000 \mathrm{fb}^{-1}$ luminosity. The higher-mass region of $H^{ \pm \pm}$can be probed with more luminosity. We find that the sensitivity reach for $H^{ \pm \pm}$in the trilepton channel is more compared to that in the tetralepton channel, as both the pair and associated production of $H^{ \pm \pm}$ contribute to the former. $H^{ \pm \pm}$up to mass $\sim 2.2 \mathrm{TeV}$ can be probed in the trilepton channel with $15 \mathrm{ab}^{-1}$ integrated luminosity. In the tetralepton channel, five events can be observed for $M_{H^{ \pm \pm}} \lesssim 1.7 \mathrm{TeV}$ with the same luminosity.

\section{ACKNOWLEDGMENTS}

M. M. acknowledges the DST-INSPIRE Grant (No. IFA14-PH-99). R. P. acknowledges the support of SAMKHYA: High Performance Computing Facility provided by Institute of Physics, Bhubaneswar.

\section{APPENDIX: BRANCHING RATIOS CALCULATION FOR THE LEPTONIC DECAYS OF $\boldsymbol{H}^{ \pm \pm}$AND $\boldsymbol{H}^{ \pm}$}

Here we expand the neutrino mass matrix in terms of the PMNS mixing angles, $C P$ phases, and the mass square differences [19]. We first consider the $H^{ \pm \pm}$decay and then the $H^{ \pm}$decay.

\section{1. $H^{ \pm \pm}$decays}

The branching ratio has the following form:

$\mathrm{BR}\left(H^{ \pm \pm} \rightarrow l_{i}^{ \pm} l_{j}^{ \pm}\right)=\frac{\Gamma_{l_{i} l_{j}}}{\sum_{k l} \Gamma_{l_{k} l_{l}}}=\frac{2}{\left(1+\delta_{i j}\right)} \frac{\left|Y_{i j}^{\nu}\right|^{2}}{\sum_{k l}\left|Y_{k l}^{\nu}\right|^{2}}$,

where

$$
\sum_{k l}\left|Y_{k l}^{\nu}\right|^{2}=\frac{1}{2 v_{\Delta}^{2}} \sum_{i} m_{i}^{2}
$$

The Yukawa and neutrino mass matrix are related as

$$
Y^{\nu}=\frac{M^{\nu}}{\sqrt{2} v_{\Delta}}=\frac{V_{\mathrm{PMNS}}^{*} m_{\mathrm{d}}^{\nu} V_{\mathrm{PMNS}}^{\dagger}}{\sqrt{2} v_{\Delta}} .
$$

Neutrino mass matrix elements can be written as a function of light neutrino masses and mixing parameters in the following forms (Here $c_{i j} \equiv \cos \theta_{i j}$ and $s_{i j} \equiv \sin \theta_{i j}$ ):

$$
\begin{aligned}
M_{11}^{\nu}= & m_{1} e^{-i \Phi_{1}} c_{12}^{2} c_{13}^{2}+m_{2} s_{12}^{2} c_{13}^{2}+m_{3} e^{i\left(2 \delta-\Phi_{2}\right)} s_{13}^{2}, \\
M_{22}^{\nu}= & m_{1} e^{-i \Phi_{1}}\left(-s_{12} c_{23}-e^{-i \delta} c_{12} s_{13} s_{23}\right)^{2}+m_{2}\left(c_{12} c_{23}-e^{-i \delta} s_{12} s_{13} s_{23}\right)^{2}+m_{3} e^{-i \Phi_{2}} c_{13}^{2} s_{23}^{2}, \\
M_{33}^{\nu}= & m_{1} e^{-i \Phi_{1}}\left(s_{12} s_{23}-e^{-i \delta} c_{12} s_{13} c_{23}\right)^{2}+m_{2}\left(-c_{12} s_{23}-e^{-i \delta} s_{12} s_{13} c_{23}\right)^{2}+m_{3} e^{-i \Phi_{2}} c_{13}^{2} c_{23}^{2}, \\
M_{12}^{\nu}= & m_{1} e^{-i \Phi_{1}} c_{12} c_{13}\left(-s_{12} c_{23}-e^{-i \delta} c_{12} s_{13} s_{23}\right)+m_{2} s_{12} c_{13}\left(c_{12} c_{23}-e^{-i \delta} s_{12} s_{13} s_{23}\right) \\
& +m_{3} e^{i\left(\delta-\Phi_{2}\right)} s_{13} c_{13} s_{23}, \\
M_{13}^{\nu}= & m_{1} e^{-i \Phi_{1}} c_{12} c_{13}\left(s_{12} s_{23}-e^{-i \delta} c_{12} c_{23} s_{13}\right)+m_{2} c_{13} s_{12}\left(-c_{12} s_{23}-e^{-i \delta} s_{12} s_{13} c_{23}\right) \\
& +m_{3} e^{i\left(\delta-\Phi_{2}\right)} s_{13} c_{13} c_{23}, \\
M_{23}^{\nu}= & m_{1} e^{-i \Phi_{1}}\left(s_{12} s_{23}-e^{-i \delta} c_{12} s_{13} c_{23}\right)\left(-s_{12} c_{23}-e^{-i \delta} c_{12} s_{13} s_{23}\right) \\
& +m_{2}\left(-c_{12} s_{23}-e^{-i \delta} s_{12} s_{13} c_{23}\right)\left(c_{12} c_{23}-e^{-i \delta} s_{12} s_{13} s_{23}\right)+m_{3} e^{-i \Phi_{2}} c_{13}^{2} s_{23} c_{23} .
\end{aligned}
$$


Following Ref. [1], we consider the following set of $3 \sigma$ variations of PMNS mixing angles and mass-squared differences:

$$
\begin{aligned}
\sin ^{2} \theta_{12} & \Rightarrow[0.273-0.379], \\
\sin ^{2} \theta_{23} & \Rightarrow[0.445-0.599]_{\mathrm{NH}},[0.453-0.598]_{\mathrm{IH}}, \\
\sin ^{2} \theta_{13} & \Rightarrow\left([1.96-2.41] \times 10^{-2}\right)_{\mathrm{NH}},\left([1.99-2.44] \times 10^{-2}\right)_{\mathrm{IH}}, \\
\Delta m_{21}^{2} & \Rightarrow[7.05-8.14] \times 10^{-5} \mathrm{eV}^{2} \\
\left|\Delta m_{31}^{2}\right| & \Rightarrow[2.41-2.60] \times 10^{-3} \mathrm{eV}^{2}(\mathrm{NH}), \\
\left|\Delta m_{31}^{2}\right| & \Rightarrow[2.31-2.51] \times 10^{-3} \mathrm{eV}^{2}(\mathrm{IH})
\end{aligned}
$$

Below, we analytically calculate the maximum value of branching ratios for different leptonic decay modes of $H^{ \pm \pm}$. For this calculation, we assume those values of the oscillation parameters that give a maximum branching ratio for a given decay mode. The set of parameters is not necessarily the same for each mode:

(1) Inverted hierarchy neutrino mass spectrum $\left(m_{3} \approx 0\right)$ : Here, due to very small mass splitting between $m_{1}$ and $m_{2}, m_{1} \approx m_{2}=m$. This gives the summation of light neutrino mass as $\sum_{j} m_{j}^{2}=2 m^{2}$. We identify different phases, for which the branching ratios in $e e, \mu \mu, e \mu$ modes are maximal.

(a) For $\phi_{1}=0$, the $(1,1)$ element of neutrino mass matrix $M_{11}^{\nu}=m c_{13}^{2}$. The branching ratio is $\operatorname{BR}\left(H^{ \pm \pm} \rightarrow e^{ \pm} e^{ \pm}\right) \approx \frac{c_{13}^{4}}{2} \approx 0.44$.

(b) For $\delta=\phi_{1}=\phi_{2}=0$, the $(2,2)$ element of neutrino mass matrix is $M_{22}^{\nu}=\left(c_{23}^{2}+s_{13}^{2} s_{23}^{2}\right) m$.
This gives the maximal branching ratio as $\operatorname{BR}\left(H^{ \pm \pm} \rightarrow \mu^{ \pm} \mu^{ \pm}\right) \approx \frac{c_{23}^{4}}{2} \approx 0.18$.

(c) For $\phi_{2}=\pi, \delta=0$, the $(1,2)$ element of neutrino mass matrix is $M_{12}^{\nu}=c_{12} c_{13} m_{1}\left(c_{23} s_{12}+\right.$ $\left.c_{12} s_{13} s_{23}\right)+c_{13} m_{2} s_{12}\left(c_{12} c_{23}-s_{12} s_{13} s_{23}\right)$. The branching ratio in $e \mu$ mode is

$$
\begin{aligned}
& \operatorname{BR}\left(H^{ \pm \pm} \rightarrow e^{ \pm} \mu^{ \pm}\right) \\
& \approx 2 c_{13}^{2} \frac{\left(\left(c_{12}^{2}-s_{12}^{2}\right) s_{13} s_{23}+2 c_{23} c_{12} s_{12}\right)^{2}}{2} \approx 0.48
\end{aligned}
$$

(2) Normal hierarchy neutrino mass spectrum $\left(m_{1} \approx 0\right)$ : The two other masses are $m_{2} \approx 0.2 m_{3}$.

(a) For $2 \delta-\phi_{2}=0$, the $(1,1)$ element of the neutrino mass matrix is $M_{11}^{\nu}=m_{2} s_{12}^{2} c_{13}^{2}+m_{3} s_{13}^{2}$, that gives $\operatorname{BR}\left(H^{ \pm \pm} \rightarrow e^{ \pm} e^{ \pm}\right) \approx 0.008$.

(b) For $\delta=\pi$ and $\phi_{2}=0$, the $(2,2)$ element of neutrino mass matrix is $M_{22}^{\nu}=c_{12}^{2} c_{23}^{2} m_{2}+c_{13}^{2} \times$ $m_{3} s_{23}^{2}+2 c_{12} c_{23} m_{2} s_{12} s_{13} s_{23}+m_{2} s_{12}^{2} s_{13}^{2} s_{23}^{2}$. Ignoring the third and fourth terms, the branching ratio in $\mu \mu$ becomes

$$
\begin{aligned}
& \operatorname{BR}\left(H^{ \pm \pm} \rightarrow \mu^{ \pm} \mu^{ \pm}\right) \\
& \approx \frac{\left(c_{12}^{2} c_{23}^{2} m_{2}+c_{13}^{2} m_{3} s_{23}^{2}\right)^{2}}{m_{3}^{2}} \approx 0.4 .
\end{aligned}
$$

(c) For $\phi_{2}=\delta=\pi$, the $(1,2)$ element of neutrino mass matrix is $M_{12}^{\nu}=c_{13} m_{3} s_{13} s_{23}+c_{13} m_{2} s_{12} \times$ $\left(c_{12} c_{23}+s_{12} s_{13} s_{23}\right)$. The branching ratio is

$$
\operatorname{BR}\left(H^{ \pm \pm} \rightarrow e^{ \pm} \mu^{ \pm}\right) \approx \frac{2\left(0.2 c_{12} c_{23} m_{3} s_{12}+s_{13} s_{23}\left(0.2 m_{3} s_{12}^{2}+m_{3}\right)\right)^{2}}{m_{3}^{2}} \approx 0.072
$$

The different ratios of branching ratio are

$$
\begin{aligned}
& \frac{\mathrm{BR}^{\max }\left(H^{ \pm \pm} \rightarrow e^{ \pm} \mu^{ \pm}\right)_{\mathrm{IH}}}{\mathrm{BR}^{\max }\left(H^{ \pm \pm} \rightarrow e^{ \pm} \mu^{ \pm}\right)_{\mathrm{NH}}} \approx \frac{\left(c_{12}^{2} s_{13} s_{23}+2 c_{23} c_{12} s_{12}-s_{12}^{2} s_{13} s_{23}\right)^{2}}{2\left(0.2 c_{12} c_{23} s_{12}+s_{13} s_{23}\left(0.2 s_{12}^{2}+1\right)\right)^{2}} \approx 6.6, \\
& \frac{\mathrm{BR}^{\max }\left(H^{ \pm \pm} \rightarrow e^{ \pm} e^{ \pm}\right)_{\mathrm{IH}}}{\mathrm{BR}^{\max }\left(H^{ \pm \pm} \rightarrow e^{ \pm} e^{ \pm}\right)_{\mathrm{NH}}} \approx \frac{c_{13}^{4}}{2\left(0.2 c_{13}^{2} s_{12}^{2}+s_{13}^{2}\right)^{2}} \approx 50, \\
& \frac{\mathrm{BR}^{\max }\left(H^{ \pm \pm} \rightarrow \mu^{ \pm} \mu^{ \pm}\right)_{\mathrm{IH}}}{\mathrm{BR}^{\max }\left(H^{ \pm \pm} \rightarrow \mu^{ \pm} \mu^{ \pm}\right)_{\mathrm{NH}}} \approx \frac{c_{23}^{4}}{2\left(c_{12}^{2} c_{23}^{2} 0.2+c_{13}^{2} s_{23}^{2}\right)^{2}} \approx 0.45 .
\end{aligned}
$$

Similarly, one can obtain such ratios for all other modes.

\section{2. $H^{ \pm}$decays}

The coupling through which $H^{ \pm}$interact with charged lepton and neutrino is

$$
Y^{+}=\cos \theta^{+} \frac{m_{d}^{\nu} V_{\mathrm{PMNS}}^{\dagger}}{v_{\Delta}} .
$$


In the above, $\theta^{+}$is the singly charged Higgs mixing angle. The branching ratio has the following form:

$$
\mathrm{BR}\left(H^{ \pm} \rightarrow l_{j}^{ \pm} \nu\right) \equiv \sum_{i=1}^{3} \mathrm{BR}\left(H^{ \pm} \rightarrow l_{j}^{ \pm} \nu_{i}\right)=\frac{X_{j}}{\sum_{i}^{3} m_{i}^{2}}, \quad\left(l_{j}=e, \mu, \tau\right),
$$

where $X_{j}$ is defined as $X_{j}=\frac{v_{\Delta}^{2}}{\cos ^{2} \theta^{+}} \sum_{i=1}^{3}\left|Y_{i j}^{+}\right|^{2}$. The $X_{1,2,3}$ has the following form:

$$
\begin{aligned}
& X_{1}=m_{1}^{2} c_{12}^{2} c_{13}^{2}+m_{2}^{2} c_{13}^{2} s_{12}^{2}+m_{3}^{2} s_{13}^{2}, \\
& X_{2}=m_{1}^{2} c_{23}^{2} s_{12}^{2}+2 \cos (\delta)\left(m_{1}^{2}-m_{2}^{2}\right) c_{12} c_{23} s_{12} s_{13} s_{23}+\left(m_{3}^{2} c_{13}^{2}+m_{2}^{2} s_{12}^{2} s_{13}^{2}\right) s_{23}^{2}+c_{12}^{2}\left(m_{2}^{2} c_{23}^{2}+m_{1}^{2} s_{13}^{2} s_{23}^{2}\right), \\
& X_{3}=m_{3}^{2} c_{13}^{2} c_{23}^{2}-2 \cos (\delta)\left(m_{1}^{2}-m_{2}^{2}\right) c_{12} c_{23} s_{12} s_{13} s_{23}+s_{12}^{2}\left(m_{2}^{2} c_{23}^{2} s_{13}^{2}+m_{1}^{2} s_{23}^{2}\right)+c_{12}^{2}\left(m_{1}^{2} c_{23}^{2} s_{13}^{2}+m_{2}^{2} s_{23}^{2}\right) .
\end{aligned}
$$

The maximum value of branching ratio for $H^{ \pm} \rightarrow l_{j}^{ \pm} \nu$ is presented below, assuming a different type of neutrino mass spectrum.

(1) Inverted Hierarchy neutrino mass spectrum $\left(m_{3} \approx 0\right)$ : Here, $m_{1} \approx m_{2}=m \Rightarrow \sum_{j} m_{j}^{2}=2 m^{2}$.

(a) $X_{1}=c_{13}^{2} m^{2}$ gives $\operatorname{BR}\left(H^{ \pm} \rightarrow e^{ \pm} \nu\right)=\frac{c_{13}^{2}}{2} \approx 0.49$. Since there is only $\theta_{13}$ dependency in $X_{1}$, and $\theta_{13}$ is very well measured, therefore $H^{ \pm} \rightarrow e^{ \pm} \nu$ decay modes have much less uncertainty.

(b) For $\delta=0, X_{2}$ has the form $X_{2}=\left(1-s_{23}^{2}\left(1+s_{13}^{2}\right)\right) m^{2}$, that gives $\operatorname{BR}\left(H^{ \pm} \rightarrow \mu^{ \pm} \nu\right)=\frac{1-s_{23}^{2}\left(1+s_{13}^{2}\right)}{2} \approx 0.3$.

(c) For $\delta=\pi, X_{3}$ has the form $X_{3}=\left(s_{23}^{2}\left(1-s_{13}^{2}\right)+s_{13}^{2}\right) m^{2}$, that gives $\operatorname{BR}\left(H^{ \pm} \rightarrow \tau^{ \pm} \nu\right)=\frac{s_{23}^{2}\left(1-s_{13}^{2}\right)+s_{13}^{2}}{2} \approx 0.3$.

Note that $H^{ \pm} \rightarrow \mu^{ \pm} \nu / \tau^{ \pm} \nu$ decay modes have nearly equal uncertainty, as both depend on $\theta_{23}$ and $\theta_{13}$.

(2) Normal hierarchy neutrino mass spectrum $\left(m_{1} \approx 0\right)$ : For this spectrum, $m_{2}=0.2 m_{3}$, which gives $\sum_{j} m_{j}^{2} \approx m_{3}^{2}$. The $C P$ phase $\delta=\pi(0)$ will maximize $X_{2}\left(X_{3}\right)$, and hence branching ratios.
(a) $X_{1} \approx m_{2}^{2} c_{13}^{2} s_{12}^{2}+m_{3}^{2} s_{13}^{2}$ gives $\operatorname{BR}\left(H^{ \pm} \rightarrow e^{ \pm} \nu\right) \approx c_{13}^{2}(0.2)^{2} s_{12}^{2}+s_{13}^{2} \approx 0.037$,
(b) $X_{2} \approx m_{3}^{2} c_{13}^{2} s_{23}^{2}$ gives $\operatorname{BR}\left(H^{ \pm} \rightarrow \mu^{ \pm} \nu\right) \approx c_{13}^{2} s_{23}^{2} \approx 0.57$,
(c) $X_{3} \approx m_{3}^{2} c_{13}^{2} c_{23}^{2}$ gives $\operatorname{BR}\left(H^{ \pm} \rightarrow \tau^{ \pm} \nu\right) \approx c_{13}^{2} c_{23}^{2} \approx 0.53$.

[1] P.F. de Salas, D. V. Forero, C. A. Ternes, M. Tortola, and J. W. F. Valle, Status of neutrino oscillations 2018: $3 \sigma$ hint for normal mass ordering and improved $C P$ sensitivity, Phys. Lett. B 782, 633 (2018).

[2] S. Weinberg, Baryon and Lepton Nonconserving Processes, Phys. Rev. Lett. 43, 1566 (1979).

[3] F. Wilczek and A. Zee, Operator Analysis of Nucleon Decay, Phys. Rev. Lett. 43, 1571 (1979).

[4] P. Minkowski, $\mu \rightarrow e \gamma$ at a rate of one out of $10^{9}$ muon decays? Phys. Lett. 67B, 421 (1977).

[5] R. N. Mohapatra and G. Senjanovic, Neutrino Mass and Spontaneous Parity Violation, Phys. Rev. Lett. 44, 912 (1980).

[6] T. Yanagida, Horizontal symmetry and masses of neutrinos, Conf. Proc. C 7902131, 95 (1979).

[7] M. Gell-Mann, P. Ramond, and R. Slansky, Complex spinors and unified theories, Conf. Proc. C 790927, 315 (1979); arXiv:1306.4669.

[8] J. Schechter and J. W. F. Valle, Neutrino masses in $S U(2) \times$ U(1) theories, Phys. Rev. D 22, 2227 (1980).

[9] K. S. Babu, C. N. Leung, and J. T. Pantaleone, Renormalization of the neutrino mass operator, Phys. Lett. B 319, 191 (1993).
[10] S. Antusch, M. Drees, J. Kersten, M. Lindner, and M. Ratz, Neutrino mass operator renormalization in two Higgs doublet models and the MSSM, Phys. Lett. B 525, 130 (2002).

[11] M. Magg and C. Wetterich, Neutrino mass problem and gauge hierarchy, Phys. Lett. B 94, 61 (1980).

[12] T. P. Cheng and L.-F. Li, Neutrino masses, mixings and oscillations in $S U(2) \times U(1)$ models of electroweak interactions, Phys. Rev. D 22, 2860 (1980).

[13] G. Lazarides, Q. Shafi, and C. Wetterich, Proton lifetime and fermion masses in an $S O(10)$ model, Nucl. Phys. B181, 287 (1981).

[14] R. N. Mohapatra and G. Senjanovic, Neutrino masses and mixings in gauge models with spontaneous parity violation, Phys. Rev. D 23, 165 (1981).

[15] R. Foot, H. Lew, X. G. He, and G. C. Joshi, Seesaw neutrino masses induced by a triplet of leptons, Z. Phys. C 44, 441 (1989).

[16] A. Arhrib, R. Benbrik, M. Chabab, G. Moultaka, M. C. Peyranere, L. Rahili, and J. Ramadan, The Higgs potential in the type II seesaw model, Phys. Rev. D 84, 095005 (2011).

[17] P. S. Bhupal Dev, D. K. Ghosh, N. Okada, and I. Saha, $125 \mathrm{GeV}$ Higgs boson and the type-II seesaw model, J. High 
Energy Phys. 03 (2013) 150; Erratum, J. High Energy Phys. 05 (2013) 049.

[18] A. G. Akeroyd and M. Aoki, Single and pair production of doubly charged Higgs bosons at hadron colliders, Phys. Rev. D 72, 035011 (2005).

[19] P. Fileviez Perez, T. Han, G.-y. Huang, T. Li, and K. Wang, Neutrino masses and the CERN LHC: Testing type II seesaw, Phys. Rev. D 78, 015018 (2008).

[20] A. Melfo, M. Nemevsek, F. Nesti, G. Senjanovic, and Y. Zhang, Type II seesaw at LHC: The roadmap, Phys. Rev. D 85, 055018 (2012).

[21] F. del Aguila and J. A. Aguilar-Saavedra, Distinguishing seesaw models at LHC with multi-lepton signals, Nucl. Phys. B813, 22 (2009).

[22] S. Chakrabarti, D. Choudhury, R. M. Godbole, and B. Mukhopadhyaya, Observing doubly charged Higgs bosons in photon-photon collisions, Phys. Lett. B 434, 347 (1998).

[23] M. Aoki, S. Kanemura, and K. Yagyu, Testing the Higgs triplet model with the mass difference at the LHC, Phys. Rev. D 85, 055007 (2012).

[24] A. G. Akeroyd and H. Sugiyama, Production of doubly charged scalars from the decay of singly charged scalars in the Higgs triplet model, Phys. Rev. D 84, 035010 (2011).

[25] E. J. Chun and P. Sharma, Search for a doubly-charged boson in four lepton final states in type II seesaw, Phys. Lett. B 728, 256 (2014).

[26] F. del Águila and M. Chala, LHC bounds on lepton number violation mediated by doubly and singly-charged scalars, J. High Energy Phys. 03 (2014) 027.

[27] S. Banerjee, M. Frank, and S. K. Rai, Higgs data confronts sequential fourth generation fermions in the Higgs triplet model, Phys. Rev. D 89, 075005 (2014).

[28] Z. Kang, J. Li, T. Li, Y. Liu, and G.-Z. Ning, Light doubly charged Higgs boson via the $W W^{*}$ channel at LHC, Eur. Phys. J. C 75, 574 (2015).

[29] Z.-L. Han, R. Ding, and Y. Liao, LHC phenomenology of type II seesaw: Nondegenerate case, Phys. Rev. D 91, 093006 (2015).

[30] Z.-L. Han, R. Ding, and Y. Liao, LHC phenomenology of the type II seesaw mechanism: Observability of neutral scalars in the nondegenerate case, Phys. Rev. D 92, 033014 (2015).

[31] K. S. Babu and S. Jana, Probing doubly charged Higgs bosons at the LHC through photon initiated processes, Phys. Rev. D 95, 055020 (2017).

[32] Y. Du, A. Dunbrack, M. J. Ramsey-Musolf, and J.-H. Yu, Type-II seesaw scalar triplet model at a $100 \mathrm{TeV} p p$ collider: Discovery and Higgs portal coupling determination, J. High Energy Phys. 01 (2019) 101.

[33] S. Antusch, O. Fischer, A. Hammad, and C. Scherb, Low scale type II seesaw: Present constraints and prospects for displaced vertex searches, J. High Energy Phys. 02 (2019) 157.

[34] M. Aaboud et al., Search for doubly charged Higgs boson production in multi-lepton final states with the ATLAS detector using proton-proton collisions at $\sqrt{s}=13 \mathrm{TeV}$, Eur. Phys. J. C 78, 199 (2018).

[35] CMS Collaboration, A search for doubly-charged Higgs boson production in three and four lepton final states at $\sqrt{s}=13 \mathrm{TeV}$, CERN Technical Report No. CMS-PASHIG-16-036, 2017, http://cds.cern.ch/record/2242956.

[36] V. Khachatryan et al. (CMS Collaboration), Study of Vector Boson Scattering and Search for New Physics in Events with Two Same-Sign Leptons and Two Jets, Phys. Rev. Lett. 114, 051801 (2015).

[37] A. M. Sirunyan et al. (CMS Collaboration), Observation of Electroweak Production of Same-Sign $W$ Boson Pairs in the Two Jet and Two Same-Sign Lepton Final State in ProtonProton Collisions at $\sqrt{s}=13 \mathrm{TeV}$, Phys. Rev. Lett. 120, 081801 (2018).

[38] S. Kanemura, K. Yagyu, and H. Yokoya, First constraint on the mass of doubly-charged Higgs bosons in the same-sign diboson decay scenario at the LHC, Phys. Lett. B 726, 316 (2013).

[39] S. Kanemura, M. Kikuchi, K. Yagyu, and H. Yokoya, Bounds on the mass of doubly-charged Higgs bosons in the same-sign diboson decay scenario, Phys. Rev. D 90, 115018 (2014).

[40] S. Kanemura, M. Kikuchi, H. Yokoya, and K. Yagyu, LHC Run-I constraint on the mass of doubly charged Higgs bosons in the same-sign diboson decay scenario, Prog. Theor. Exp. Phys. 2015, 51B02 (2015).

[41] M. Mitra, S. Niyogi, and M. Spannowsky, Type-II seesaw and multilepton signatures at hadron colliders, Phys. Rev. D 95, 035042 (2017).

[42] D. K. Ghosh, N. Ghosh, I. Saha, and A. Shaw, Revisiting the high-scale validity of type-II seesaw model with novel LHC signature, Phys. Rev. D 97, 115022 (2018).

[43] M. Aaboud et al. (ATLAS Collaboration), Search for doubly charged scalar bosons decaying into same-sign $W$ boson pairs with the ATLAS detector, Eur. Phys. J. C 79, 58 (2019).

[44] J. Abdallah et al. (DELPHI Collaboration), Search for doubly charged Higgs bosons at LEP-2, Phys. Lett. B 552, 127 (2003).

[45] J.-F. Shen and Z.-X. Li, Doubly charged Higgs bosons pair production through $W W$ fusion at high-energy $e^{+} e^{-}$linear colliders, Europhys. Lett. 111, 31001 (2015).

[46] S. Blunier, G. Cottin, M. A. Díaz, and B. Koch, Phenomenology of a Higgs triplet model at future $e^{+} e^{-}$colliders, Phys. Rev. D 95, 075038 (2017).

[47] J. Cao and X.-Y. Tian, Doubly and singly charged Higgs pair production at high-energy $e^{+} e^{-}$linear colliders, Int. J. Mod. Phys. A 31, 1650056 (2016).

[48] Y.-C. Guo, C.-X. Yue, and Z.-C. Liu, The signatures of doubly charged leptons in future linear colliders, J. Phys. G 44, 085004 (2017).

[49] P. Agrawal, M. Mitra, S. Niyogi, S. Shil, and M. Spannowsky, Probing the type-II seesaw mechanism through the production of Higgs bosons at a lepton collider, Phys. Rev. D 98, 015024 (2018).

[50] P. S. B. Dev, S. Khan, M. Mitra, and S. K. Rai, Doublycharged Higgs boson at a future electron-proton collider, Phys. Rev. D 99, 115015 (2019).

[51] P.S. Bhupal Dev and Y. Zhang, Displaced vertex signatures of doubly charged scalars in the type-II seesaw and its left-right extensions, J. High Energy Phys. 10 (2018) 199. 
[52] Y. Cai, T. Han, T. Li, and R. Ruiz, Lepton number violation: Seesaw models and their collider tests, Front. Phys. 6, 40 (2018).

[53] A. Abada et al., HE-LHC: The high-energy large hadron collider, Eur. Phys. J. Special Topics 228, 1109 (2019).

[54] M. Cepeda et al. (HL/HE WG2 Group), Higgs physics at the HL-LHC and HE-LHC, arXiv:1902.00134.

[55] C. Patrignani et al. (Particle Data Group), Review of particle physics, Chin. Phys. C 40, 100001 (2016).

[56] L. Lavoura and L.-F. Li, Making the small oblique parameters large, Phys. Rev. D 49, 1409 (1994).

[57] E. J. Chun, H. M. Lee, and P. Sharma, Vacuum stability, perturbativity, EWPD and Higgs-to-diphoton rate in type II seesaw models, J. High Energy Phys. 11 (2012) 106.

[58] J. Garayoa and T. Schwetz, Neutrino mass hierarchy and Majorana $C P$ phases within the Higgs triplet model at the LHC, J. High Energy Phys. 03 (2008) 009.

[59] E. J. Chun, K. Y. Lee, and S. C. Park, Testing Higgs triplet model and neutrino mass patterns, Phys. Lett. B 566, 142 (2003).

[60] N. Aghanim et al. (Planck Collaboration), Planck 2018 results. VI. Cosmological parameters, arXiv:1807.06209.

[61] M. Muhlleitner and M. Spira, A note on doubly charged Higgs pair production at Hadron colliders, Phys. Rev. D 68, 117701 (2003).

[62] T. Ghosh, S. Jana, and S. Nandi, Neutrino mass from Higgs quadruplet and multicharged Higgs searches at the LHC, Phys. Rev. D 97, 115037 (2018).

[63] P. S. B. Dev, R. N. Mohapatra, and Y. Zhang, Probing the Higgs sector of the minimal left-right symmetric model at future hadron colliders, J. High Energy Phys. 05 (2016) 174.

[64] D. Borah, B. Fuks, D. Goswami, and P. Poulose, Investigating the scalar sector of left-right symmetric models with leptonic probes, Phys. Rev. D 98, 035008 (2018).

[65] A. Aboubrahim and P. Nath, Naturalness, the hyperbolic branch, and prospects for the observation of charged Higgs bosons at high luminosity LHC and $27 \mathrm{TeV}$ LHC, Phys. Rev. D 98, 095024 (2018).
[66] A. Crivellin, M. Ghezzi, L. Panizzi, G. M. Pruna, and A. Signer, Low- and high-energy phenomenology of a doubly charged scalar, Phys. Rev. D 99, 035004 (2019).

[67] T. B. de Melo, F. S. Queiroz, and Y. Villamizar, Doubly charged scalar at the high-luminosity and high-energy LHC, Int. J. Mod. Phys. A 34, 1950157 (2019).

[68] A. Alloul, N. D. Christensen, C. Degrande, C. Duhr, and B. Fuks, FeynRules 2.0: A complete toolbox for tree-level phenomenology, Comput. Phys. Commun. 185, 2250 (2014).

[69] J. Alwall, R. Frederix, S. Frixione, V. Hirschi, F. Maltoni, O. Mattelaer, H. S. Shao, T. Stelzer, P. Torrielli, and M. Zaro, The automated computation of tree-level and next-to-leading order differential cross sections, and their matching to parton shower simulations, J. High Energy Phys. 07 (2014) 079.

[70] R. D. Ball, V. Bertone, S. Carrazza, L. Del Debbio, S. Forte, A. Guffanti, N. P. Hartland, and J. Rojo (NNPDF Collaboration), Parton distributions with QED corrections, Nucl. Phys. B877, 290 (2013).

[71] T. Sjostrand, S. Mrenna, and P.Z. Skands, A brief introduction to PYTHIA 8.1, Comput. Phys. Commun. 178, 852 (2008).

[72] J. de Favereau, C. Delaere, P. Demin, A. Giammanco, V. Lemaître, A. Mertens, and M. Selvaggi (DELPHES 3 Collaboration), DELPHES 3, A modular framework for fast simulation of a generic collider experiment, J. High Energy Phys. 02 (2014) 057.

[73] R. Brun and F. Rademakers, ROOT: An object oriented data analysis framework, Nucl. Instrum. Methods Phys. Res., Sect. A 389, 81 (1997).

[74] A. M. Sirunyan et al. (CMS Collaboration), Performance of the CMS muon detector and muon reconstruction with proton-proton collisions at $\sqrt{s}=13 \mathrm{TeV}$, J. Inst. 13, P06015 (2018).

[75] A. M. Sirunyan et al. (CMS Collaboration), Search for additional neutral MSSM Higgs bosons in the $\tau \tau$ final state in proton-proton collisions at $\sqrt{s}=13 \mathrm{TeV}$, J. High Energy Phys. 09 (2018) 007.

[76] B. Fuks, M. Nemevšek, and R. Ruiz, Doubly charged Higgs boson production at hadron colliders, arXiv:1912.08975. 